\title{
Macrophage migration inhibitory factor mediates peripheral nerve injury-induced hypersensitivity by curbing dopaminergic descending inhibition
}

\author{
Xian Wang ${ }^{1,6}$, Shaolei $\mathrm{Ma}^{2,6}$, Haibo $\mathrm{Wu}^{1,6}$, Xiaofeng Shen ${ }^{1,6}$, Shiqin $\mathrm{Xu}^{1}$, Xirong Guo ${ }^{3}$, Maria L Bolick ${ }^{4}$, \\ Shizheng $\mathrm{Wu}^{5}$ and Fuzhou Wang ${ }^{1,4}$
}

Our previous works disclosed the contributing role of macrophage migration inhibitory factor (MIF) and dopaminergic inhibition by lysine dimethyltransferase G9a/Glp complex in peripheral nerve injury-induced hypersensitivity. We herein propose that the proinflammatory cytokine MIF participates in the regulation of neuropathic hypersensitivity by interacting with and suppressing the descending dopaminergic system. The lumbar spinal cord (L-SC) and ventral tegmental area (VTA) are two major locations with significant upregulation of MIF after chronic constriction injury (CCI) of the sciatic nerve, and they display time-dependent changes, along with a behavioral trajectory. Correspondingly, dopamine (DA) content shows the reverse characteristic change to MIF with a time-dependent curve in post-surgical behavior. The levels of both MIF and DA are reversed by the MIF tautomerase inhibitor ISO-1, and a negative relationship exists between MIF and DA. The reversed role of ISO-1 also affects tyrosine hydroxylase expression. Furthermore, CCI induces Th promoter CpG site methylation in the L-SC and VTA areas, and this effect could be abated by ISO-1 administration. G9a/SUV39H1 and H3K9me2/H3K9me3 enrichment within the Th promoter region following $\mathrm{CCI}$ in the L-SC and VTA was also decreased by ISO-1. In cultured dopaminergic neurons, rMIF enhanced the recruitment of G9a and SUV39H1, followed by an increase in $\mathrm{H} 3 \mathrm{~K} 9 \mathrm{me} 2 / \mathrm{H} 3 \mathrm{~K} 9 \mathrm{me}$. These molecular changes correspondingly exhibited alterations in Th promoter CpG site methylation and pain behaviors. In summary, MIF functions as a braking factor in curbing dopaminergic descending inhibition in peripheral nerve injury-induced hypersensitivity by mediating Th gene methylation through G9a/SUV39H1-associated H3K9 methylation.

Experimental \& Molecular Medicine (2018) 50, e445; doi:10.1038/emm.2017.271; published online 16 February 2018

\section{INTRODUCTION}

Neuropathic pain is a debilitating experience for patients, usually resulting from a direct lesion or disease affecting the somatosensory system and causing a heavy burden for patients, health care providers and society. ${ }^{1}$ Despite recent advances, the underlying mechanisms that are responsible for neuropathic pain arising from nerve injury remain incompletely understood, and the currently available treatments are still unsatisfactory. The monoamine neurotransmitter dopamine plays a crucial role in nociceptive transmission within various supraspinal brain regions. ${ }^{2,3}$ Additionally, dopaminergic descending projection to the spinal cord also critically participates in abnormal pain nociception, ${ }^{4,5}$ as well as enhancing injury- induced central sensitization mediated by other monoamines. ${ }^{6}$ In experimental ${ }^{7-9}$ and clinical settings, ${ }^{7}$ descending dopaminergic inhibition pathways as intervention targets in neuropathic pain treatment have attracted increasing attention.

Macrophage migration inhibitory factor (MIF) is a pleiotropic cytokine, and it was first described in 1966 as a proinflammatory cytokine participating in macrophage motility inhibition. ${ }^{8}$ MIF usually functions as an inflammatory cytokine, and it is widely involved in a variety of diseases that have inflammatory components, such as infections, cancer, autoimmune diseases and metabolic disorders. ${ }^{9-11}$ Further, MIF also acts as an anterior pituitary hormone secreted in tandem with glucocorticoids to counter-regulate stress responses, ${ }^{12}$ and

\footnotetext{
${ }^{1}$ Department of Anesthesiology, Obstetrics and Gynecology Hospital, Affiliated to Nanjing Medical University, Nanjing, China; ${ }^{2}$ Department of Critical Care Medicine, The First Affiliated Hospital of Nanjing Medical University, Nanjing, China; ${ }^{3}$ Institute of Pediatrics, Obstetrics and Gynecology Hospital, Affiliated to Nanjing Medical University, Nanjing, China; ${ }^{4}$ Group of Neuropharmacology and Neurophysiology, Division of Neuroscience, The Bonoi Academy of Science and Education, Chapel Hill, NC, USA and ${ }^{5}$ Department of Neurology, Qinghai Provincial People's Hospital, Xining, China

${ }^{6}$ These authors contributed equally to this work.

Correspondence: Dr S Wu, Department of Neurology, Qinghai Provincial People's Hospital, Xining 811600, China. or Dr F Wang, Department of Anesthesiology Obstetrics and Gynecology Hospital, Affiliated to Nanjing Medical University, Nanjing 210004, China.

E-mail: wushizheng2005@hotmail.com or fred.wang@basehq.org

Received 10 May 2017; revised 25 August 2017; accepted 12 September 2017
} 
it is a redox-sensitive enzyme that affects cell growth and survival. ${ }^{13}$ Recently, several lines of evidence have suggested a contributing role for MIF in neuropathic pain development. Widely expressed throughout the central and peripheral nervous systems, especially within regions participating in sensory transmission, MIF content prominently increases after nerve injury, reaching a striking $\sim 1000$-fold greater content than other algogenic cytokines, such as IL-6, IL- $1 \beta$ and TNF-a. ${ }^{14}$ In addition, recombinant MIF (rMIF) injection was sufficient to evoke pain-like behaviors in naïve mice, and MIF was involved in stress-induced neuropathic pain enhancement. ${ }^{15}$ In contrast, MIF null mice failed to develop, while MIF inhibition attenuated pain-like hypersensitivity in sciatic nerve-sparing nerve injury or chronic constriction injury models. ${ }^{15,16}$ Although possible mechanisms of MIF in response to nerve injury have been suggested, including sensitizing nociceptive neurons, evoking algogenic gene transcription in microglia (e.g., IL-6, IL-1 $\beta$, TNF-a, CCL-2 and iNOS), and activating central sensitization-related signaling pathways (e.g., ERK, p38, Src, Jab-1 and PI3K), ${ }^{15,17}$ the exact role of MIF in neuropathic pain is far from clearly defined.

Sporadic evidence has suggested that MIF is involved in dopamine metabolism to detoxify dopamine cytotoxicity, possibly deriving from its enzymatic function. ${ }^{18-20}$ Additionally, one report showed that MIF is involved in the generation of depression-like symptoms by potentiating the effects of IFN$\gamma$ on dopamine metabolism. ${ }^{21}$ Thus, we strongly speculate that there is possible regulation of MIF for dopamine in pain regulation, combined with the contributing role of MIF, as well as the descending dopamine pathway, in neuropathic pain, $3,15,16,22$ and we suppose that the proinflammatory cytokine MIF participates in the regulation of neuropathic hypersensitivity by interacting with and suppressing the dopaminergic system.

Considering our previous findings that dopaminergic epigenetic inhibition resulted from tyrosine hydroxylase $(T h)$ gene CpG site methylation alteration via the methyltransferase G9a/ Glp complex contributing to dopamine downregulation in the ventral tegmental area (VTA) following nerve injury, ${ }^{23}$ we hereby hypothesize that MIF might play an essential role in the underlying mechanisms of peripheral nerve injury-induced neuropathic pain through epigenetic regulation of the descending dopamine inhibitory pathway, with possible involvement of histone methyltransferase (HMTase) G9a/Glp and SUV39H1, as well as $\mathrm{H} 3 \mathrm{~K} 9$ methylation, and Th promoter methylation is verified as well.

\section{MATERIALS AND METHODS}

\section{Experimental subjects}

With the approval of the Institutional Ethics Committee, all of the experiments were conducted under the guidance of Ethical Guidelines for Investigations of Experimental Pain in Conscious Animals. Male C57BL/6 mice weighting 20-25 g with an age of 7-9 weeks were used for behavioral tests. The peri-surgical treatment of the animals was reported previously. ${ }^{16}$ Briefly, the animals were housed on a reverse $12: 12 \mathrm{~h}$ light/dark cycle with the housing temperature at $23 \pm 1{ }^{\circ} \mathrm{C}$.
After randomization using numbers generated by the online software QuickCalcs\# (GraphPad, San Diego, CA), the test sessions occurred in a quiet room between 1000 and 1800 hours. To reduce stress before each test, the mice were placed in a test box to habituate for $10 \mathrm{~min}$, and then the test sessions were conducted. Animal model establishment and the following molecular detection procedures were performed by different experimenters to achieve blinding. Each mouse was used only once and was euthanized at the end of the behavioral experiment by administrating a lethal dose of pentobarbital.

\section{Testing drugs and antibodies}

The MIF inhibitor ISO-1 (S,R)-3-(4-hydroxyphenyl)-4,5-dihydro-5isoxazole acetic acid (Merck KGaA, Darmstadt, Germany) was used in this study to inhibit the catalytic site of MIF, which has been demonstrated to markedly reduce its biological function. ${ }^{24,25}$ To test the role of MIF on Th gene $\mathrm{CpG}$ methylation in vitro, mouse recombinant MIF (rMIF) was purchased from R\&D Systems (Minneapolis, MN, USA). Recombinant G9a and SUV39H1 were purchased from Active Motif (Carlsbad, CA, USA). 5-Aza (5-aza-2'deoxycytidine) is a well-known DNA methyltransferase inhibitor. ${ }^{26}$ Histone deacetylases (HDACs) result in a compact and inactive chromatin structure that has been reported to regulate histone modifications, ${ }^{27}$ while TSA is a well-characterized chemical inhibitor of HDACs. ${ }^{28}$ Chaetocin is an SUV39H1-specific inhibitor. ${ }^{29}$ 5-Aza, TSA and chaetocin were purchased from Sigma (St. Louis, MO, USA), and HDAC1 was obtained from Reaction Biology Corp. (Malvern, PA, USA). In addition, BIX 01294 is a specific and potent G9a inhibitor. ${ }^{24}$ Anti-dopamine transporter (DAT)-Saporin (Advanced Targeting Systems, San Diego, CA, USA) specifically targets and destroys dopaminergic neurons and is a type of dopaminergic immunotoxin. ${ }^{30}$ Recombinant G9a, SUV39H1 and anti-DAT-Saporin were dissolved in PBS, while the other drugs were dissolved in 10\% DMSO, and the corresponding solvent was used as vehicle control.

Antibodies against G9a, H3K9me2 and H3K9me3 were obtained from Millipore (Bedford, MA), antibodies against MIF, tyrosine hydroxylase (TH), DAT, SUV39H1, HP1, histone $\mathrm{H} 3, \beta$-actin and $\beta$-tubulin, as well as normal IgG, horseradish-peroxidase conjugated secondary antibodies, were purchased from Santa Cruz Biotechnology (Santa Cruz, CA, USA), and all of them were employed for western blot or chromatin immunoprecipitation (ChiP) assay.

\section{Chronic constriction injury of the sciatic nerve}

We established chronic neuropathic pain with chronic constriction injury (CCI) of the sciatic nerve, as first described by Bennett and Xie. $^{31}$ In brief, under anesthesia with isoflurane, the biceps femoris was bluntly dissected, the unilateral sciatic nerve was exposed, and four loose ligations were applied proximal to the trifurcation of the sciatic nerve to cause visible retardation of the epineural vasculature. After surgery, the incision was sutured and iodine solution was topically used to prevent infection. All of the animals were observed closely after they were returned to the home cage. For sham surgeries, the unilateral sciatic nerve was only exposed but not ligated.

\section{Pain behavior measurements}

Pain behaviors during the 70-day observation period following CCI were detected using von Frey filaments (Stoelting, Chicago, IL, USA), a Randall-Selitto analgesymeter (Model, 37215; UGO Basile, Italy), and a hot plate (Eddy's Hot Plate; Naugra Export, Ambala Cantt, Haryana, India), respectively. 
A series of von Frey filaments $(0.4,0.7,0.16,0.40,0.60,1.0,1.4,2.0$, $4.0,6.0,8.0,10,15,26$, and $60 \mathrm{~g}$ ) were vertically applied to the central plantar of the hind paw to measure allodynia, as described in our previous study. ${ }^{32}$ In brief, the weakest filament of $0.4 \mathrm{~g}$ was initially applied to evoke a withdrawal behavior for a maximum of $10 \mathrm{~s}$. If the paw withdrew or the mouse flinched, the same hair was again used $60 \mathrm{~s}$ later; when a response was lacking, the next greatest force was presented. If the mouse withdrew its paw twice consecutively with the same filament force, no further tests were needed. Absolute threshold, expressed as $50 \%$ of the withdrawal threshold, was determined after adjusting to a Gaussian integral psychometric function using the maximum-likelihood method.

Mechanical hyperalgesia was measured with the Randall-Selitto analgesymeter by previously described methods. ${ }^{33}$ Briefly, increasing pressure at a linear rate of $10 \mathrm{~g} \mathrm{~s}^{-1}$ was applied to the hind paw center using the Randall-Selitto paw pressure analgesymeter with a maximum of $150 \mathrm{~g}$ to avoid potential tissue damage. Each trial was repeated three times with a 15-min interval, and the mechanical pressure $(\mathrm{g})$ at which the animal withdrew its paw was recorded.

Thermal hyperalgesia was determined with a hot plate with the temperature set at $55 \pm 0.1^{\circ} \mathrm{C}$, and paw withdrawal latency was recorded according to the number of seconds required for the characteristic lifting or licking of the hind paw after the thermal stimulus. The maximal stimulation duration was set at $22 \mathrm{~s}$ as a cutoff value to avoid tissue injury. Each animal test was repeated three times with a 15-min interval, and the thermal withdrawal latency (sec) was recorded.

\section{Western blot detection}

For tissue western blot analysis, region-specific tissue, including the dorsal root ganglion (DRG), lumbar spinal cord L5-6 (L-SC), VTA, nucleus accumbens (NAc), prefrontal cortex $(\mathrm{PFc})$, locus caeruleus (LC), nigra-striatum (NS) and hippocampus (HC), was separated and minced into small pieces and then was suspended in lysis buffer $(4 \%$ SDS, $0.004 \%$ bromophenol blue, $20 \%$ glycerol, Tris- $\mathrm{HCl}, 10 \%$ 2-mercaptoethanol, $\mathrm{pH}$ 6.8, containing $50 \mathrm{~mm}$ PMSF, $6 \mathrm{M}$ urea, $50 \mathrm{~mm} \mathrm{Na}_{3} \mathrm{VO}_{4}$, Sigma) for protein extraction. For western blot analysis of neurons, total proteins were prepared from lysed cells using radioimmunoprecipitation assay lysis buffer (RIPA). The Bradford method ${ }^{34}$ was used to determine the concentration of total protein. Protein samples were adjusted to a concentration of $10 \mu \mathrm{g} \mathrm{ll}^{-1}$ in protein lysis buffer and LDS sample buffer in volumes sufficient to perform all of the immunoblot analyses and to confirm equal protein content after fractionation by SDS-PAGE and transfer to PVDF membranes, blocking with $5 \%$ nonfat milk in PBS containing $1 \%$ Tween-20 (PBST), and incubating with various indicated antibodies overnight at $4{ }^{\circ} \mathrm{C}$. The membranes were washed with PBST, incubated with species-appropriate secondary antibodies. Enhanced chemiluminescence was used to visualize the bands. Finally, protein levels were normalized to the signal obtained with the corresponding housekeeping protein, and then the protein expression was expressed as a relative density compared to normal or sham densities.

\section{Microdialysate sample collection}

For time-dependent detection of MIF or monoamine contents, each mouse had a guide cannula stereotaxically placed (AG-4, Eicom, Japan) at a specific nerve location according to the atlas of Paxinos and Frankin $^{35}$ with sodium pentobarbital $\left(50 \mathrm{mg} \mathrm{kg}^{-1}\right.$, i.p.) anesthesia, including L-SC (L5/L6 spinal cord), VTA (anterior $-3.2 \mathrm{~mm}$, lateral $+0.75 \mathrm{~mm}$, ventral $-4.5 \mathrm{~mm}$ from bregma), NAc (anterior $+1.2 \mathrm{~mm}$, lateral $+1.0 \mathrm{~mm}$, ventral $-5.0 \mathrm{~mm}$ from bregma), $\mathrm{PFc}$ (anterior $+2.0 \mathrm{~mm}$, lateral $+0.5 \mathrm{~mm}$, ventral $-3.0 \mathrm{~mm}$ from bregma), LC (anterior $-5.45 \mathrm{~mm}$, lateral $+1.20 \mathrm{~mm}$, ventral $-3.65 \mathrm{~mm}$ ), NS (anterior $+0.2 \mathrm{~mm}$, lateral $+2.0 \mathrm{~mm}$, ventral $-2.6 \mathrm{~mm}$ from bregma) and $\mathrm{HC}$ (anterior $-2.2 \mathrm{~mm}$, lateral $+1.3 \mathrm{~mm}$, ventral $-1.5 \mathrm{~mm}$ from bregma). The guide cannula was secured on the neighboring fascia with sutures and was buried under the skin. Sodium penicillin 10000 IU was administered intramuscularly to prevent infection. Each mouse was only used for one nerve location. The mice were allowed to recover for $24 \mathrm{~h}$ after stereotaxic surgery.

On day 0 and days $1,2,4,7,14,21,35,49$ and 70 post CCI, microdialysate samples were collected after the insertion of a probe (A-I-4-2, Eicom) carrying an active dialysis membrane $(2 \mathrm{~mm}$ in length; inner diameter, $0.20 \mathrm{~mm}$; outer diameter, $0.22 \mathrm{~mm}$; cutoff value, $50 \mathrm{kDa}$ ) constructed from hemicellulose dialysis tubing. The probe's inlet was continuously perfused with artificial cerebrospinal fluid (145 mM NaCl, $2.7 \mathrm{~mm} \mathrm{KCl}, 1.2 \mathrm{mM} \mathrm{CaCl}, 1.0 \mathrm{mM} \mathrm{MgCl}_{2}$, $2.0 \mathrm{mM} \mathrm{Na}_{2} \mathrm{HPO}_{4} ; \mathrm{pH} 7.4$ ) at a constant rate of $1.0 \mathrm{ml} \mathrm{min}^{-1}$ using a $1.0-\mathrm{ml}$ gas-tight syringe (Hamilton, Reno, NV, USA). Dialysate samples were collected during 20 min of sampling using outlet tubing connected to a microfraction collector (CMA Microdialysis).

After completion of the microdialysis experiments, the mice were administered a lethal dose of pentobarbital $\left(100 \mathrm{mg} \mathrm{kg}^{-1}\right)$ and decapitated. The probe position was verified by the histological examination, and only data from mice with correct probe placement were used.

\section{Enzyme-linked immunosorbent assay}

For time-dependent MIF content detection in L-SC, VTA, NAc, PFc, LC, NS and HC over 70 days following CCI, region-specific microdialysate was used for MIF detection with ELISA. However, for MIF content detection with or without ISO-1 in L-SC and VTA on postinjury day 21, region-specific tissue homogenate was first centrifuged, and the resulting supernatant was prepared for ELISA. The detailed ELISA procedure was according to the manufacturer's instructions. Ninety-six well ELISA plates were coated with rabbit anti-mouse MIF (Invitrogen, Carlsbad, CA, USA). Unspecific binding was blocked with PBS containing $1 \% \mathrm{BSA} / 5 \%$ sucrose/ $0.05 \% \mathrm{NaHN}_{3}$. After washing three times, recombinant mouse MIF test samples and standard sera were added to the wells and incubated for $2 \mathrm{~h}$. Biotinylated polyclonal rabbit anti-mouse MIF was used as the detection antibody, and streptavidin-HRP was used as the second-step reagent. Color was visualized with 3,3',5,5'-tetramethylbenzidine (Sigma-Aldrich, St. Louis, MO, USA), and absorbance was measured at $450-620 \mathrm{~nm}$ against standard curves.

\section{High-performance liquid chromatography}

Monoamine contents in normal, sham, CCI or CCI+ISO-1 mice at post-injury day 14 or 21 , as well as time-dependent monoamine contents in CCI mice over the 70 days post injury, were analyzed with high-performance liquid chromatography (HPLC) using microdialysates or tissue-derived supernatants. The main operative conditions for HPLC consisted of the following: $3 \mathrm{~mm}$ Ultrasphere ODS column; mobile phase: $0.15 \mathrm{~mm} \mathrm{NaH}{ }_{2} \mathrm{PO}_{4} / 0.01 \mathrm{~mm}$ octyl sodium sulfate/ $0.5 \mathrm{~mm} \mathrm{EDTA} / 12.5 \%$ methanol at a rate of $1 \mathrm{ml} \mathrm{min}^{-1}$; model 590 delivery pump (Waters Associates, Milforal); and a detector (ESA Coulochem mod. 5100A with a dual electrode analytical cell (Mod. 5011)). 


\section{Intrathecal and intraventricular catheterization}

Intrathecal or Intraventricular catheters were implanted 1 week before nerve injury. Intrathecal catheters were implanted as described in our previous study. ${ }^{36}$ In brief, after sterilization, a midline incision was performed in the cephalic-cervical area, and then the paravertebral muscles were dissected. With the help of a surgical microscope, a hole $(1 \times 1 \mathrm{~mm})$ was drilled manually through the cisternal membrane to the dura. The catheter (ALZET Osmotic Pumps, Cupertino, CA, USA) was inserted $2.5 \mathrm{~mm}$ caudal from the dural slit, fixed with a drop of tissue glue (Histoacry; B. Braun, Tuttingen, Germany), and further immobilized on the paravertebral fascia. Finally, sodium penicillin 10000 IU (Shanghai AoBopharmtech, Shanghai, China) was administered intramuscularly to prevent infection.

Intraventricular catheters were implanted as previously shown. ${ }^{23}$ Briefly, after sterilization, a hole was stereotaxically drilled at lateral 1.0 and $0.5 \mathrm{~mm}$ posterior to the bregma in the skull, and a $26-\mathrm{G}$ stainless steel infusion cannula was initially placed $2.0 \mathrm{~mm}$ below the skull surface to the right ventricle. Then, a stylus was inserted to maintain patency, and the catheter was secured to the skull with a drop of tissue glue. Finally, a total dose of $30 \mathrm{mg} \mathrm{kg}^{-1}$ of ibuprofen was given orally in drinking water for antinociception for at least 3 days postoperatively, while sodium penicillin $10000 \mathrm{IU}$ and dexamethasone $0.4 \mathrm{mg}$ were administered given intramuscularly and subcutaneously to prevent infection and to reduce brain swelling, respectively. If neurological deficits were shown after surgery, either intrathecally or intraventricularly catheterized mice were excluded from further analysis. All intrathecal and intraventricular administered drugs were injected using a microsyringe in a volume of $5 \mu \mathrm{l}$ for more than $30 \mathrm{~s}$, followed by $10 \mu \mathrm{l}$ of saline to flush the catheter dead space.

\section{Bisulfite sequencing}

Th promoter $\mathrm{CpG}$ island methylation profiles were detected with bisulfite sequencing. Genomic DNA from fresh tissue or primary cultured dopaminergic neurons were extracted with a DNeasy kit according to the manufacturer's protocol (Qiagen, Hilden, Germany) and were quantified using a spectrophotometer (Biophotometer Plus, Eppendorf, Germany). Then, DNA (1 mg) was treated with bisulfite using a Zymo DNA Methylation Kit (Zymo Research, Orange). Bisulfite-treated DNA was eluted in $10 \mathrm{ml}$ volumes with $1 \mathrm{ml}$ used for each PCR. Primers were biotinylated to convert the PCR products to single-stranded DNA templates. The PCR products $(10 \mathrm{ml})$ were sequenced by pyrosequencing using the PSQ96 HS System (Biotage, Kungsgatan, Sweden), based on the manufacturer's protocol. The methylation status of each locus was individually detected as a T/C SNP with QCpG software. ${ }^{37}$

\section{VTA dopaminergic neuron culture in vitro}

Dopaminergic neurons were cultured according to the methods described by Bayer et al. ${ }^{38}$ with a few modifications. Briefly, the ventral tegmental area tissue from embryonic day 13 mice was dissected and collected, transferred to $0.1 \%$ trypsin and $0.05 \%$ DNAse and incubated at $37^{\circ} \mathrm{C}$ with $5 \% \mathrm{CO}_{2}$ for $20 \mathrm{~min}$; then it was washed in DNAse, homogenized, and centrifuged at $200 \mathrm{~g}$, resuspended in medium (DMEM, 10\% FCS, 1\% P/S, 1.5\% HEPES, 1\% Sodium Pyruvate) and then plated on 96-well dishes coated with poly-L-lysine. After $90 \mathrm{~min}$, the medium was changed to neurobasal medium (neurobasal (Gibco 21103-049) with 1\% P/S, 2\% B27, 1.25\% and $0.5 \mathrm{~mm}$ L-glutamine). The purity of dopaminergic neuron cultures was established by staining as described for dopaminergic neuron marker dopamine receptor.

\section{ChiP and real-time PCR}

ChIP assay was performed as described by others ${ }^{39}$ with some modifications using a ChiP Express kit (Active Motif). Briefly, for tissue ChiP assay, minced L-SC or VTA was cross-linked with $1 \%$ formaldehyde, followed by the addition of $2.5 \mathrm{M}$ glycine to a final concentration of $0.125 \mathrm{M}$ for $10 \mathrm{~min}$ to terminate the reaction at $4{ }^{\circ} \mathrm{C}$. Tissue samples were centrifuged and washed repeatedly to obtain a single cell suspension. After centrifugation, the resuspended pellet was lysed in FA Lysis buffer, and the collected nuclei were subjected to sonication, while for neuron ChiP assay, cells were crosslinked with $1 \%$ formaldehyde, followed by the addition of $125 \mathrm{~mm}$ glycine for 5 min to terminate the reaction and lysed in SDS lysis buffer with protease inhibitor, and the collected nuclei were subjected to sonication as well.

Obtained samples were sonicated to generate DNA fragments in lengths of 200-1000 bp and were immunoprecipitated with antibodies against G9a, SUV39H1, HP1, H3K9me2 and H3K9me3 and antibody against IgG as a negative control, following preincubation with protein $\mathrm{G}$ magnetic beads overnight at $4{ }^{\circ} \mathrm{C}$, and $1 \%$ sonicated chromatin as an input control. The resulting antibody-chromatin complex was pulled down by protein $G$ magnetic beads and washed. Then, the cross-links were reversed and followed by proteinase-K treatment. Next, the released DNA fragments were purified with a Qiagen DNA extraction kit. The DNA fragments were detected by real-time PCR using a SYBR Green kit (Takara, Tokyo, Japan) with the following primers: G9a, 5'-GAGGTGTACTGCATAGATGCC-3' (forward) and 5'-CAGACGGCTCTGCTCCAGGGC-3' (reverse); SUV39H1, 5'-AA GGATGCAGTGTGTGTTGC-3' (forward) and 5'-CCTGTTCGCG GATCTTTTTA-3' (reverse); and HP1, 5'-GAAAGAAGACC AAGAGGACAGC-3' (forward) and 5' ${ }^{\prime}$-TTGTTTCACCCTC CTTCATCT-3' (reverse).

After each PCR run, dissociation curves were generated to ensure that a single product of the appropriate length was amplified. The levels of indicated targets in the Th promoter were calculated using the $2^{-\triangle \Delta C T}$ method. ${ }^{40}$

\section{Statistical analyses}

The pain behavioral trajectories were modeled with the fixed effect analysis method in SAS software, version 9.4 (SAS Institute Inc., Cary, NC, USA), and were analyzed with non-linear regression. All other data were presented as the means \pm standard errors of mean (s.e.m.'s) and were analyzed using GraphPad Prism software, version 5.0 (GraphPad Software Inc., San Diego, CA, USA) or PASW Statistics software, version 18.0 (IBM Co., Armonk, NY, USA). When there were two testing groups, Student's $t$-test was used to analyze the intergroup difference. One-way ANOVA, followed by Bonferroni's post hoc tests for multiple comparisons, was used when necessary. In addition, correlation analysis was performed for MIF and monoamine contents in L-SC and VTA. Pain intensity in response to von Frey filaments following different inhibitors or activators was quantified using the area under the curve (AUC) method. All of the reported $P$ values were two-sided, and a $P$-value $<0.05$ was considered to be statistically significant.

\section{RESULTS}

Establishment of chronic neuropathic pain with CCI of the unilateral sciatic nerve

The pain behaviors of sham and CCI mice were depicted for 70 days and were presented with a fixed effect model, which helped to determine whether distinct inter- and intra-group 

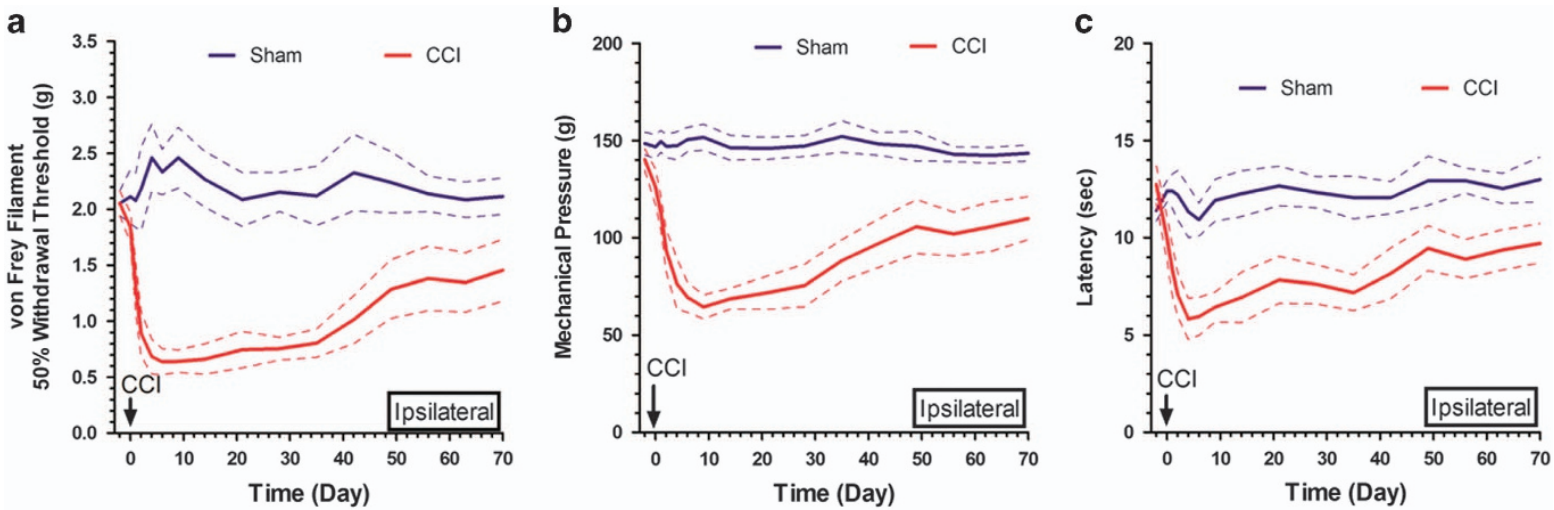

Figure 1 Effect of chronic constriction injury (CCl) of sciatic nerve on ipsilateral pain behaviors. A fixed effect model of pain behaviors, including ipsilateral 50\% withdrawal threshold, mechanical pressure, and thermal withdrawal latency in sham and $\mathrm{CCl}$ mice, is depicted over the 70-day post injury observational period (a-c); the solid and dotted lines represent the means of pain thresholds and their $95 \%$ confidence intervals, respectively). $N=15$ in each group.

behavioral differences existed among different groups of animals. It was suggested that either the ipsilateral $50 \%$ withdrawal threshold, mechanical pressure $(\mathrm{g})$, or thermal withdrawal latency $(\mathrm{sec})$ decreased drastically following CCI; nearly peaked on days 14-21 post injury; and progressively recovered thereafter. However, these values were still lower than those of the sham-operated mice at the end of the 70-day post-injury observational period (Figure $1 \mathrm{a}-\mathrm{c}$ ). At the same time, no significant differences were observed with regard to contralateral pain behaviors, including the 50\% withdrawal threshold, mechanical pressure (g) and thermal withdrawal latency (sec) (Supplementary Figure $1 \mathrm{a}-\mathrm{c}$ ). These results indicated that CCI of the sciatic nerve successfully mimicked characteristic allodynia, mechanical hyperalgesia or thermal hyperalgesia following peripheral nerve injury and established chronic neuropathic pain. According to these pain behavior trajectories, MIF and monoamine contents, Th gene CpG site methylation levels, and pain behavior changes following different regulators were detected on day 14 or 21 post CCI.

\section{MIF expression and monoaminergic neurotransmitter content following CCI}

To examine the possible role of MIF in the monoaminergic descending inhibitory pathway, we initially detected MIF expression by western blot and the monoaminergic neurotransmitter content by HPLC on day 14 post CCI in eight cerebrospinal regions, namely, DRG, L-SC, VTA, NAc, PFc, LC, NS and HC. Compared with normal and sham mice, CCI mice displayed a significant increase in MIF protein expression in L-SC and VTA but not in the other cerebrospinal regions (Figure $2 \mathrm{a}$ and $\mathrm{b}$ ), suggesting that L-SC and VTA were two locations where MIF upregulation functioned, while the dopamine content decreased prominently in all of the aforementioned locations in CCI mice compared to normal and sham mice (Figure 2c). In addition, the NE content in CCI mice increased only in DRG, L-SC, and LC and was not changed in the other locations (Figure 2d); however, the 5-HT content remained unchanged in all eight regions in CCI mice compared to normal and sham mice (Figure 2e).

This expression discrepancy with regard to nerve location among the three monoamines suggested that they might play different roles after peripheral nerve injury, closely related to specific expression regions. However, the relationship between MIF upregulation in L-SC/VTA and broad dopamine downregulation following peripheral injury remains unclear. In addition, because LC is the major producer of norepinephrine in the central nervous system, increased norepinephrine in DRG and L-SC might be part of the LC-centered descending spinal pathway, ${ }^{41}$ but their involvement in CCI cannot be excluded.

Given these queries and the need to exclude the potential influence of specific observational time points, MIF expression and monoaminergic neurotransmitter content were further detected time-dependently with ELISA and HPLC on days 0,1 , $2,4,7,14,21,35,49$ and 70 post CCI within each nerve location. We originally found that only MIF and dopamine content showed time-dependent tendencies. MIF expression quickly increased in the L-SC and VTA at several days post injury, peaked on day 14 post CCI, gradually decreased afterward, and was still higher than baseline (Figure 3a). In contrast, the dopamine content in the L-SC and VTA abruptly decreased several days post injury, peaked on days 14-21 post CCI, gradually recovered subsequently, and was still lower than baseline (Figure 3b). There were no significant changes in MIF and dopamine content in the other nerve locations, including $\mathrm{NAc}, \mathrm{PFc}, \mathrm{LC}, \mathrm{NS}$ and $\mathrm{HC}$, and no NE or 5-HT content changes within individual nerve locations throughout the 70day observation period (Supplementary Figure $2 \mathrm{a}-\mathrm{d}$ ). These results again emphasize that L-SC and VTA are the two key locations with MIF upregulation and dopamine downregulation. Interestingly, the time-dependent expression curves of MIF and dopamine post CCI were consistently with the postsurgical behavioral trajectory shown in Figure 1. 
a
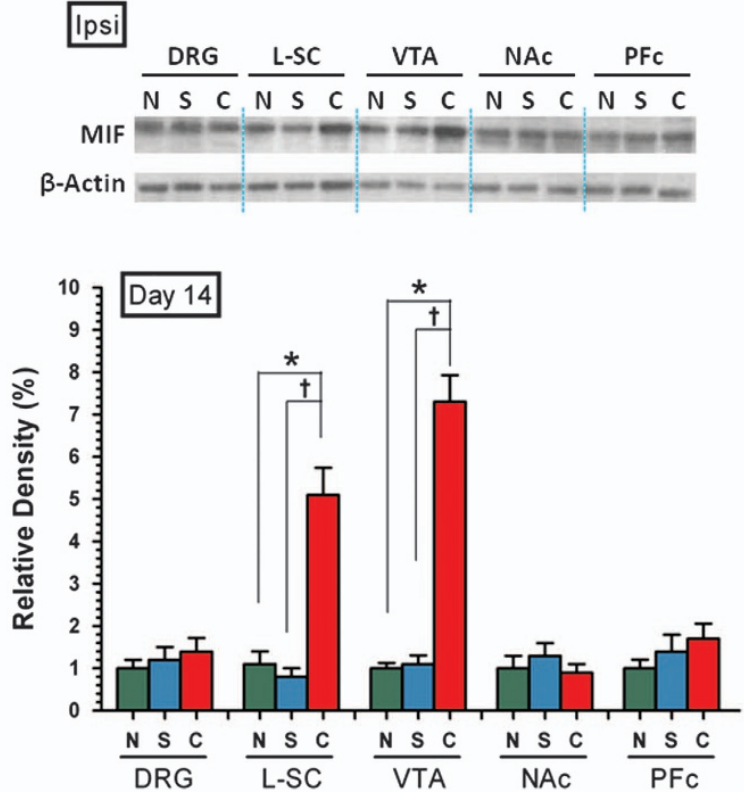

C

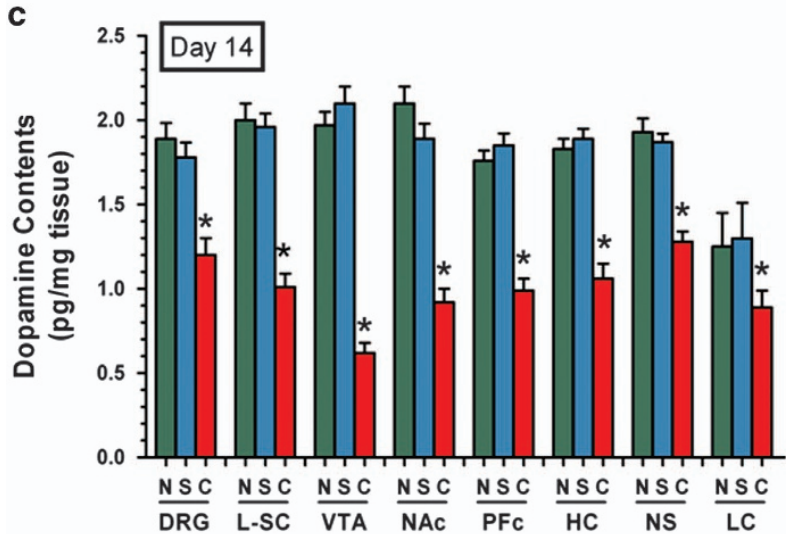

e

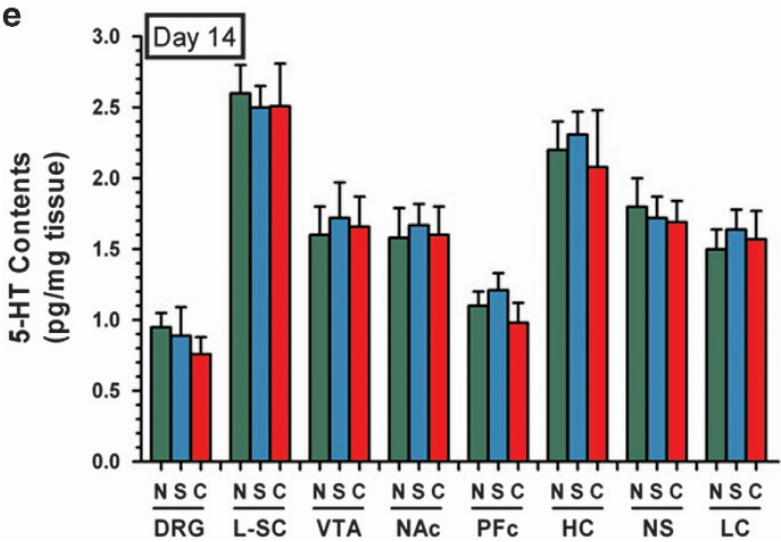

b
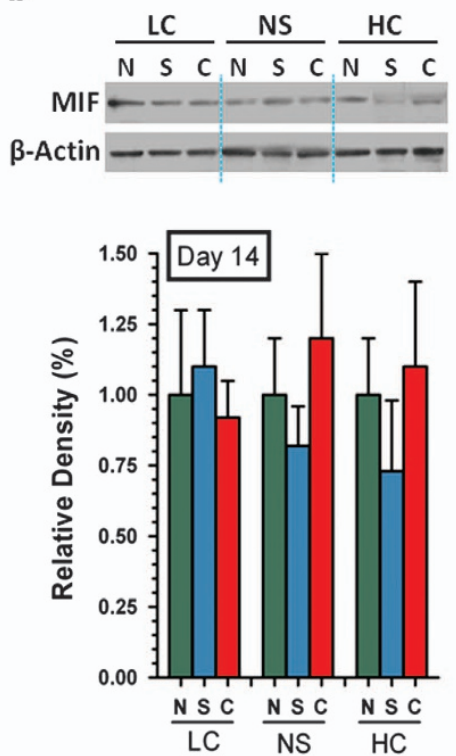

d

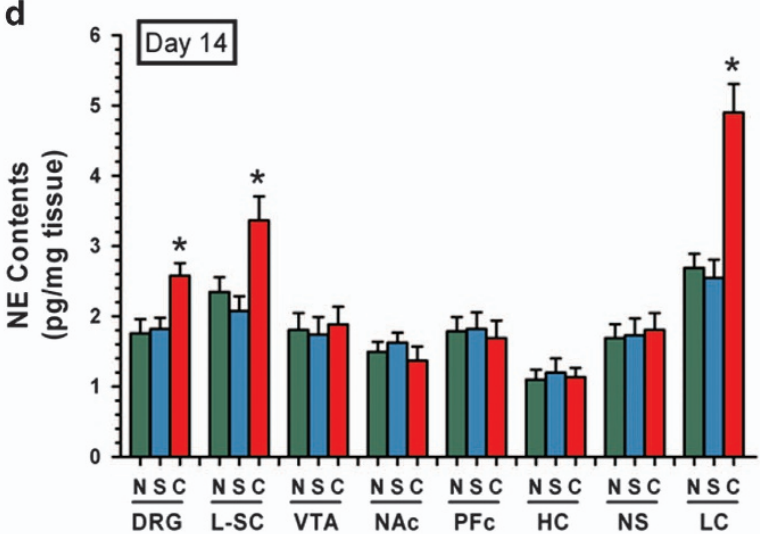

Figure 2 Migration inhibitory factor (MIF) expression and monoaminergic neurotransmitter content in normal, sham, and chronic constriction injury (CCl) mice at post-injury day 14. In normal, sham and $\mathrm{CCl}$ mice, MIF expression and monoaminergic neurotransmitter content, including dopamine, NE and 5-HT, were detected by western blot and HPLC, respectively, in eight cerebrospinal regions including DRG, L-SC, VTA, NAc, PFc, LC, NS and HC at post-injury day 14. Representative western blot gel images and relative density of MIF expression in normal, sham and $\mathrm{CCl}$ mice are presented in $\mathbf{a}$ and $\mathbf{b}\left({ }^{*} P<0.05\right.$ vs normal mice; ${ }^{\dagger} P<0.05$ vs sham mice; $\beta$-actin was used as loading control; $n=15$ in each group). Dopamine, NE and 5-HT content is shown in c-e ( $n=15$ in each group). Data are shown as the means \pm s.e.m.'s and were analyzed with one-way ANOVA.C, CCI; DRG, dorsal root ganglion; HC, hippocampus; LC, locus caeruleus; L-SC, lumbar spinal cord L5-6; N, normal; NAc, nucleus accumbens; NS, nigra-striatum; PFc, prefrontal cortex; S, sham; VTA, ventral tegmental area. 
a

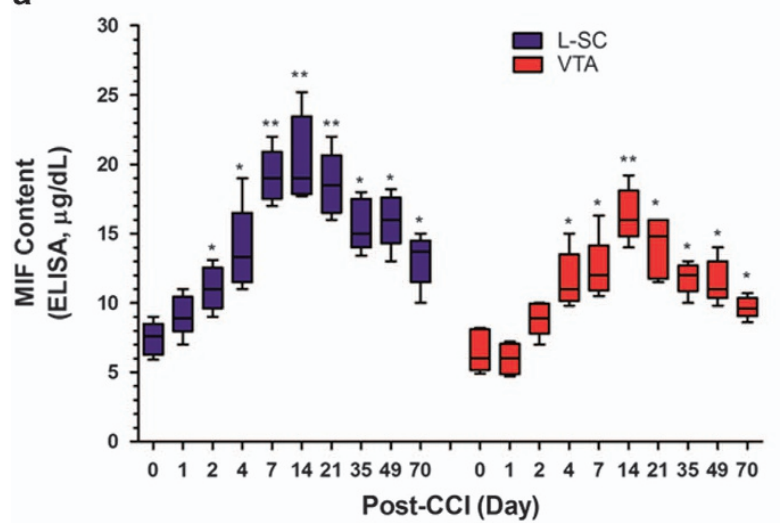

b

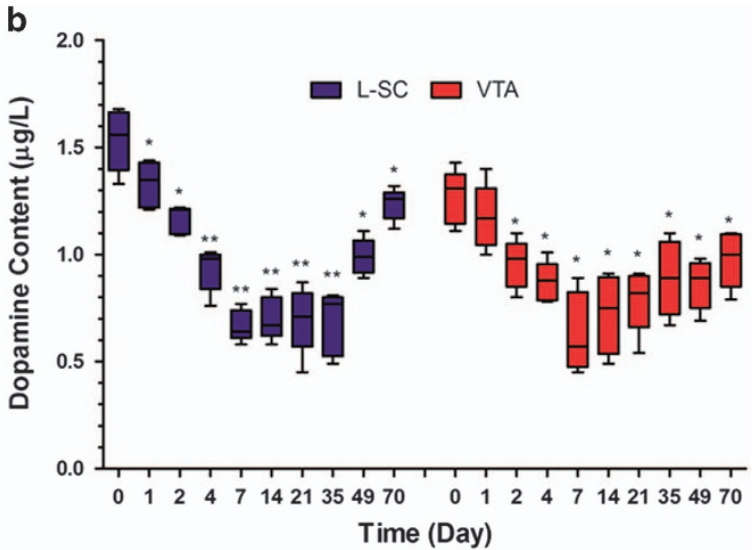

Figure 3 Migration inhibitory factor (MIF) and dopamine content in L-SC and VTA in CCI mice over the 70-day observation period. MIF and dopamine content in microdialysate obtained from L-SC and VTA were detected with ELISA and HPLC, respectively, on days 0, 1, 2, 4, 7, 14, 21, 35, 49 and 70 post CCI. Time-dependent MIF content upregulation in L-SC and VTA, as well as inversed dopamine content downregulation, are shown in $\mathbf{a}$ and $\mathbf{b}\left({ }^{*} P<0.05,{ }^{*} P<0.01\right.$ vs day 0$)$. Data are shown as the means \pm s.e.m.'s and were analyzed with one-way ANOVA. $N=15$ in each group. CCI, chronic constriction injury; L-SC, lumbar spinal cord L5-6; VTA, ventral tegmental area.

Effects of ISO-1 on MIF and monoamine content in L-SC and VTA following CCI

Based on the aforementioned observations, first, only MIF and dopamine showed time-dependent tendencies post-nerve injury; second, the characteristic expression changes in MIF and dopamine coincided with the pain behavior trajectory post CCI; third, both MIF upregulation and dopamine downregulation were only located in L-SC and VTA. Therefore, we further investigated the potential relationship of MIF and dopamine in L-SC and VTA with the specific MIF tautomerase inhibitor ISO-1. Both in vivo and in vitro studies have demonstrated a dose-dependent inhibitory effect of ISO-1 on MIF tautomerase activity. ${ }^{42}$ ISO-1 $30 \mu$ g per day was i.t. or i.c.v. administered for 2 weeks from day 7 post nerve injury. The dose of ISO-1 was selected based on our previous work, wherein ISO-1 improved the mechanical threshold and thermal latency after CCI injury with efficacy of $40>30>20 \mu \mathrm{g}=10 \mu \mathrm{g}=$ DMSO. ${ }^{16}$ Then, MIF and monoamine contents in L-SC and VTA were detected with ELISA and HPLC at post-injury day 21, respectively. As expected, ISO-1 significantly reversed upregulated MIF expression following CCI in L-SC after i.t. (Figure 4a) or in VTA after i.c.v. treatment (Figure 4d). In addition, ISO-1 prominently restored decreased dopamine content following CCI in L-SC (Figure 4b) and VTA (Figure 4e), showing a similar turnover effect. However, ISO-1 had no influence on NE or 5-HT expression following CCI, in either L-SC or VTA. Correlation analysis consistently displayed an obvious non-linear negative relationship between MIF expression and dopamine content in L-SC (Figure 4c) and VTA (Figure 4f) in sham, CCI and CCI +ISO-1 mice.

Effects of ISO-1 on TH and DAT expression, as well as pain behaviors following CCI

Because dopamine content depends on $\mathrm{TH}$ and DAT, the former is a rate-limiting enzyme for dopamine synthesis, while the latter is a type of presynaptic membrane protein for rapid reuptake of dopamine, regulating both dopamine content and function at synaptic clefts. ${ }^{43}$ To explore the possible relationship of MIF and TH/DAT, we detected TH/DAT expression in DRG, L-SC, VTA, NAc and PFc at post-injury day 14 with western blot. The results indicated obviously lower $\mathrm{TH}$ expression in L-SC and VTA of CCI mice compared to normal or sham mice (Figure 5a), coincident with the MIF expression changes shown in Figure 2a, while no DAT expression change was observed in the aforementioned nerve locations in normal, sham and CCI mice (Figure 5b). Both findings indicate that there might an inherent relationship between MIF and $\mathrm{TH}$, which requires further confirmation; however, it was ascertained that presynaptic membrane DAT is not involved in dopamine content regulation following CCI.

To further verify the causal relationship between MIF and $\mathrm{TH}$, the MIF inhibitor ISO-1 $30 \mu \mathrm{g}$ per day was i.t. or i.c.v. injected for 2 weeks from day 7 post nerve injury. $\mathrm{TH}$ expression in L-SC and VTA, as well as pain behaviors, was observed with western blot and responses to von-Frey filaments. The results showed that ISO-1 significantly restored CCI-induced $\mathrm{TH}$ downregulation in L-SC and VTA (Figure 5c), while either i.t. or i.c.v. ISO-1 could prominently improve CCI-evoked allodynia (Figure $5 \mathrm{~d}$ and e).

\section{Th promoter CpG island methylation with or without ISO-1 following CCI}

Based on the contributing role of MIF for dopamine/TH, combined with the dopaminergic descending inhibitory function in neuropathic pain, we originally proposed the following regulation scenario: nerve injury induced MIF upregulation in L-SC and VTA, which downregulated TH expression, decreasing central dopamine content, inhibiting the dopaminergic descending inhibitory pathway, and finally contributing to central sensitization. However, the mechanism underlying central TH downregulation post CCI remains unclear. A previous study demonstrated that the DNA methylation status of CpG islands in the Th promoter underlies the specificity of 
a

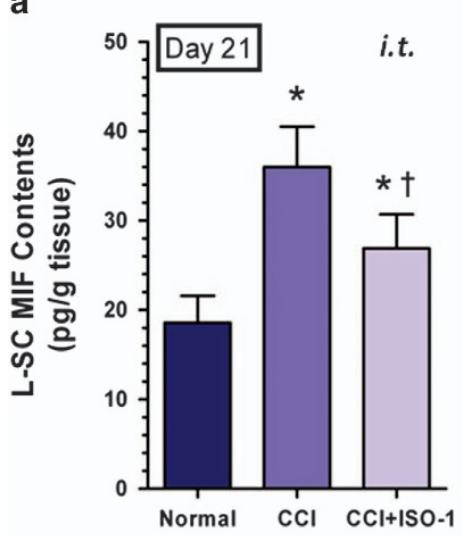

d

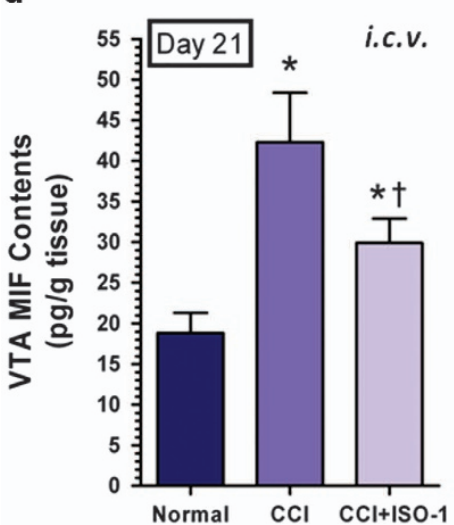

b

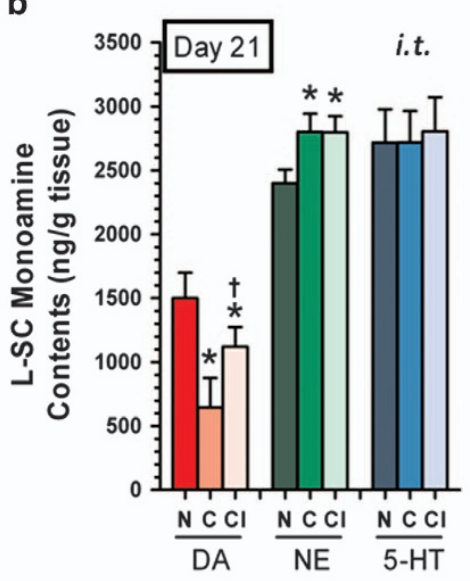

e

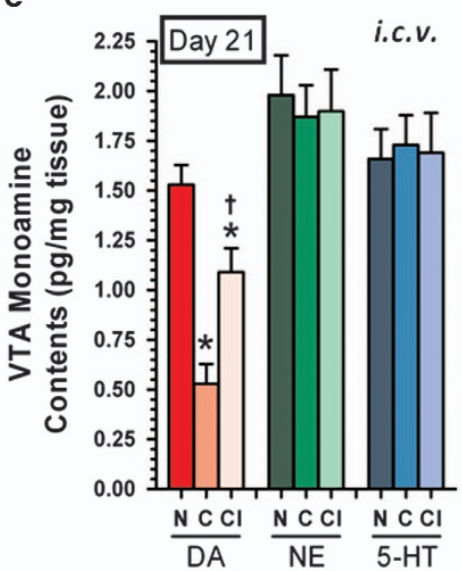

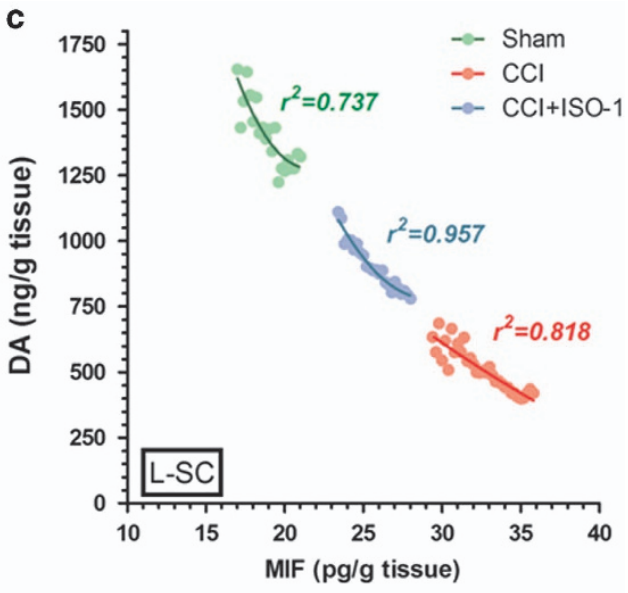

f

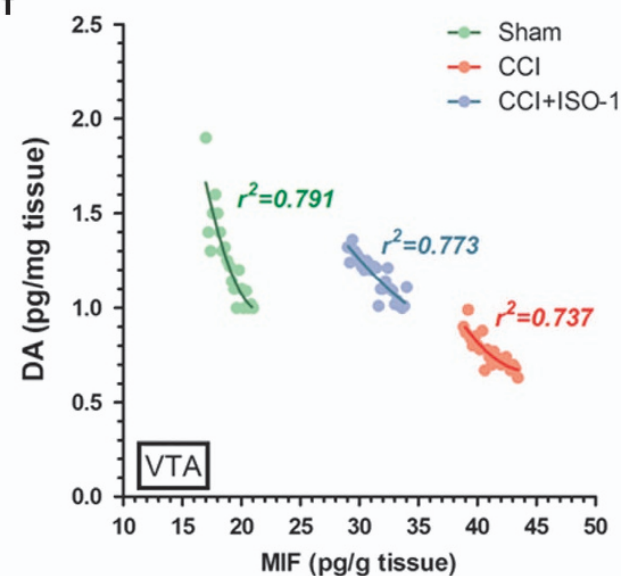

Figure 4 Effects of ISO-1 on migration inhibitory factor (MIF) and monoamine content in L-SC and VTA following chronic constriction injury (CCI). The MIF inhibitor ISO-1 was administered i.t. or i.c.v. for 2 weeks from day 7 post-nerve injury. In L-SC and VTA, the MIF and monoamine content was detected with ELISA and HPLC, respectively, at post-injury day 21. The turnover effect of i.t. ISO-1 for MIF and dopamine in L-SC is shown in $\mathbf{a}$ and $\mathbf{b}\left({ }^{*} P<0.05\right.$ vs normal mice; ${ }^{\dagger} P<0.05$ vs CCI mice). In VTA, a similar turnover phenomenon of i.c.v. ISO-1 regarding MIF and dopamine was also observed (d, e, ${ }^{\star} P<0.05$ vs normal mice; ${ }^{\dagger} P<0.05$ vs CCI mice). Data are shown as the means \pm s.e.m.'s and were analyzed with one-way ANOVA. $N=15$ in each group. Then, correlation analysis clearly showed an obvious non-linear, negative relationship between MIF and dopamine in L-SC and VTA in sham, CCl, or CCl+ISO-1 mice (c, f). C, CCl; Cl, CCl +ISO-1; L-SC, lumbar spinal cord L5-6; N, normal; VTA, ventral tegmental area.

TH expression in neural stem cells, ${ }^{44}$ and more evidence showed that the MIF genetic polymorphism was involved in $\mathrm{CpG}$ island hypermethylation profiles in carcinogenesis, ${ }^{45}$ suggesting a possible pro-methylation effect of MIF on the target gene. Thus, we suggest that nerve injury-associated high MIF expression might induce high Th promoter CpG island methylation to control $\mathrm{TH}$ transcription and finally decrease central dopamine content.

Accordingly, we initially detected Th gene promoter $\mathrm{CpG}$ island methylation levels in DRG, L-SC, VTA, NAc and PFc on day 21 following CCI with bisulfite sequencing (BiSS). The results showed that the $T h \mathrm{CpG}$ island methylation level was markedly increased post-CCI in only L-SC and VTA but not in other areas (Figure $6 \mathrm{a}-\mathrm{c}$ ). To further explore the possible role of MIF in Th CpG island methylation, ISO-1 $30 \mu \mathrm{g}$ per day was i.t. or i.c.v. injected for 2 weeks from day 7 post nerve injury, Th CpG island methylation in L-SC and VTA was detected on day 21 post CCI. Interestingly, ISO-1 prominently decreased
CCI-induced Th CpG island hypermethylation in L-SC and VTA (Figure 6d and e), consistent with our speculation that, in CCI-induced neuropathic pain, the Th gene is hypermethylated in L-SC and VTA, and this hypermethylation status is closely related to MIF upregulation.

\section{Relationship of MIF and Th CpG island methylation in cultured dopaminergic neurons}

To further understand the role of MIF in Th CpG island hypermethylation, cultured dopaminergic neurons from day 7 in vitro were treated with ascending doses of mouse rMIF $(0,0.5,5,50$ and $500 \mu \mathrm{M})$ for $30 \mathrm{~min}$ at $37^{\circ} \mathrm{C}$, and $\mathrm{Th}$ promoter $\mathrm{CpG}$ island methylation profiles were evaluated with BiSS. The results showed that the Th gene methylation percentage increased in a dose-dependent manner $(500>50>5>0.5>0 \mu \mathrm{M})$ with increasing concentrations of rMIF (Figure $7 \mathrm{a}$ and $\mathrm{b}$ ). At the same time, to test the role of ISO-1 in rMIF-induced Th gene hypermethylation, 
a
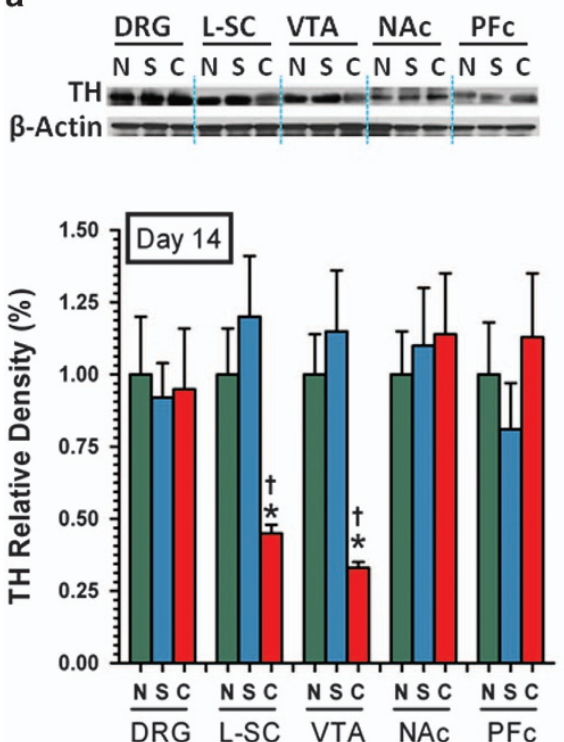

d

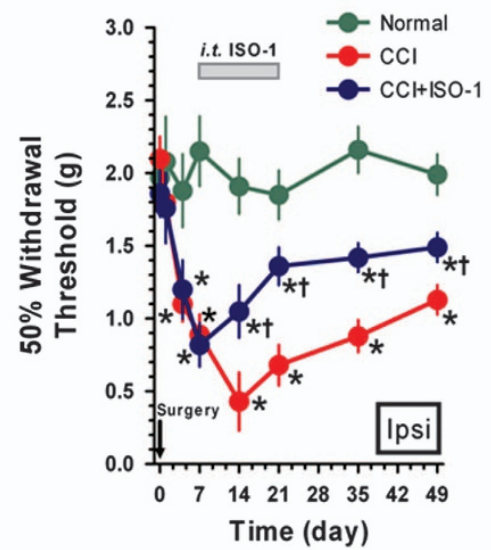

b
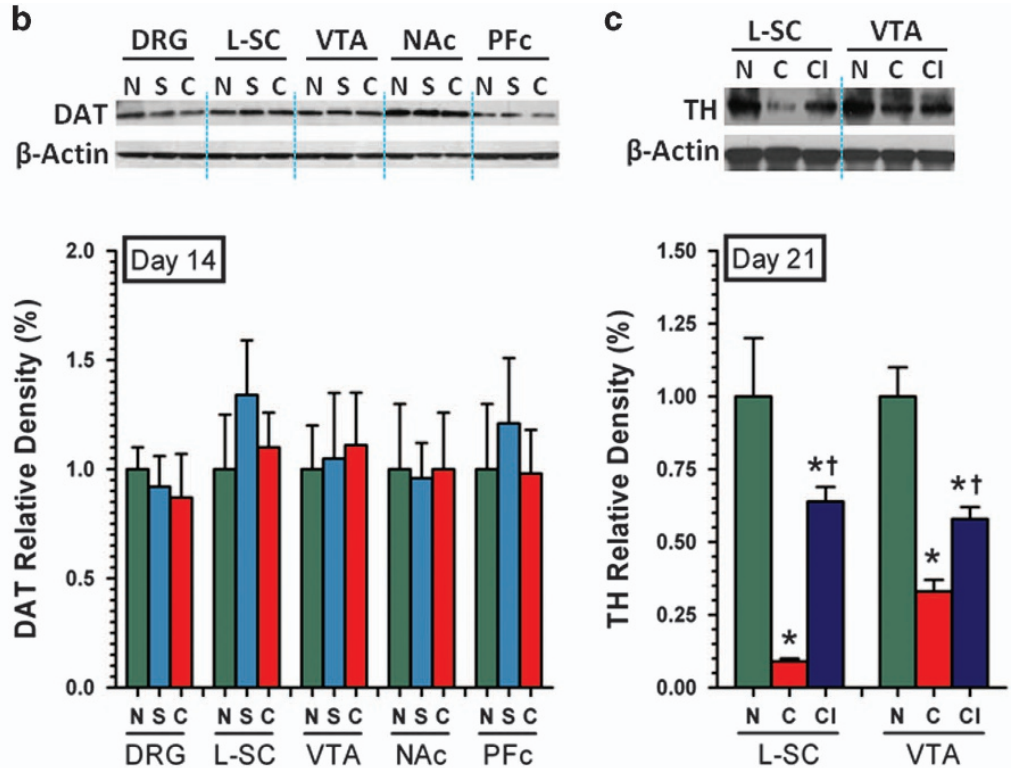

e

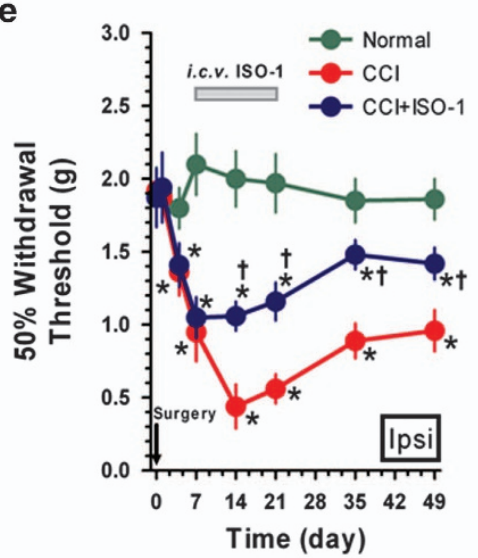

Figure 5 Effects of ISO-1 on TH and DAT expression, as well as pain behaviors following chronic constriction injury (CCI). TH and DAT expression in DRG, L-SC, VTA, NAc and PFc at post-injury day 14 in normal, sham and CCl mice was detected with western blot. Representative western blot gel images and the relative density of TH and DAT are presented in $\mathbf{a}$ and $\mathbf{b}$, ${ }^{*} P<0.05$ vs normal mice; ${ }^{\dagger} P<0.05$ vs sham mice). Then, ISO-1 was i.t. or i.c.v. injected for 2 weeks from day 7 post nerve injury, and TH expression in L-SC/VTA, as well as pain behaviors in normal, $\mathrm{CCl}$ and $\mathrm{CCl}+\mathrm{ISO}-1$ mice, was detected with western blot and von-Frey filaments, respectively. Representative western blot gel images and the relative density of TH expression in L-SC/VTA are presented in $c$ ( $P<0.05$ vs normal mice; ${ }^{\dagger} P<0.05$ vs $\mathrm{CCI}$ mice). Pain behaviors in response to von-Frey filaments are depicted for 49 days post i.t. or i.c.v. ISO-1 treatment (d, e). Data are shown as the means \pm s.e.m.'s and were analyzed with two-way ANOVA, $n=15$ in each group. $\mathrm{C}, \mathrm{CCl} ; \mathrm{Cl}, \mathrm{CCl}+\mathrm{ISO}-1$; DAT, dopamine transporter; N, normal; S, sham; TH, tyrosine hydroxylase.

vehicle (10\% DMSO), rMIF $500 \mu \mathrm{M}$, or rMIF $500 \mu \mathrm{M}+\mathrm{ISO}-1$ $40 \mu \mathrm{M}$ (ED ${ }_{50}$ of ISO-1 in vitro for MIF inhibition in neurons) was applied to cultured dopaminergic neurons for $30 \mathrm{~min}$ at $37^{\circ} \mathrm{C}$. Interestingly, the BiSS results suggested that ISO-1 significantly decreased rMIF-induced CpG island hypermethylation status (Figure $7 \mathrm{c}$ and $\mathrm{d}$ ). These data again emphasize MIF's role in mediating Th gene methylation alterations in dopaminergic neurons. When the direct demethylation agent 5-Aza $(15 \mu \mathrm{M})$ was incubated with dopaminergic neurons, rMIF-induced Th gene methylation was totally blocked (Figure $7 \mathrm{e}$ and $\mathrm{f}$ ); indicating that $T h$ gene methylation was the potential molecular mechanism of MIF-induced TH expression. Herein, the dose of ISO-1 was based on its dose-dependent inhibitory effects on MIF tautomerase activity and MIF levels in vitro $(100>10>1>0.1 \mu \mathrm{M}) .^{16}$

G9a, SUV39H1, HP1, H3K9me2, and H3K9me3 expression in L-SC and VTA

Considering that DNA methylation is one characteristic of epigenetic regulation in post-mitotic neurons, combined with the phenomenon of MIF regulation of Th promoter CpG island methylation, we propose that peripheral injury-induced MIF high expression might contribute to the development of central hypersensitivity via epigenetic dopaminergic inhibition because DNA methylation and histone modification are two 


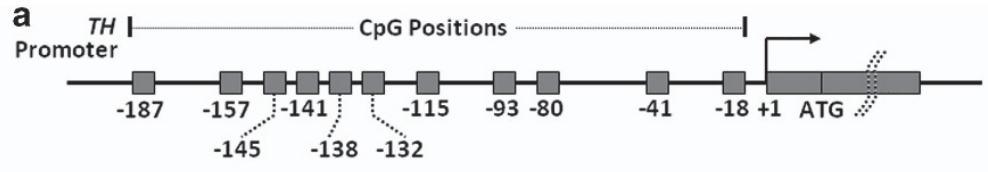

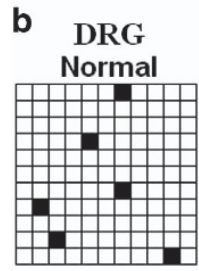

CCI

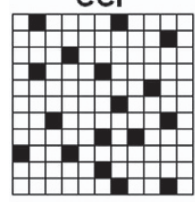

L-SC Normal

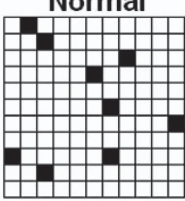

$\mathrm{CCl}$

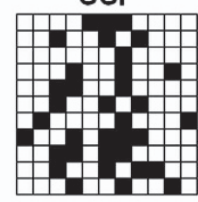

VTA

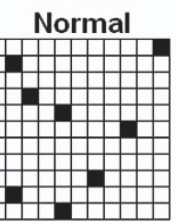

$\mathrm{CCl}$

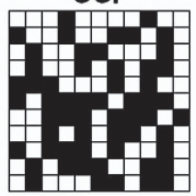

NAc

Normal

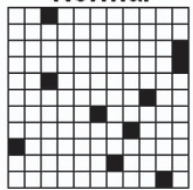

$\mathrm{CCl}$

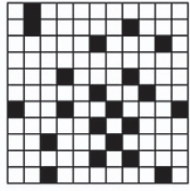

PFc Normal

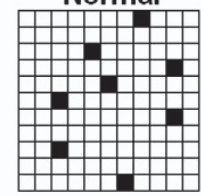

$\mathrm{CCl}$

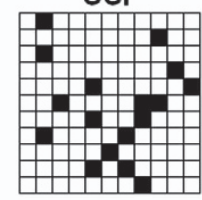

C

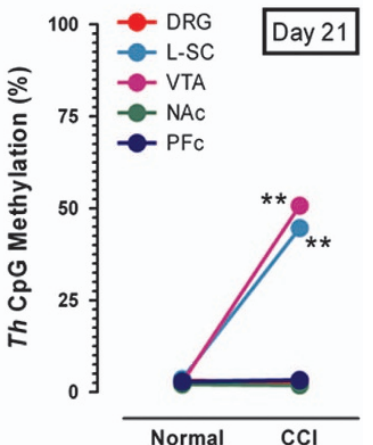

d

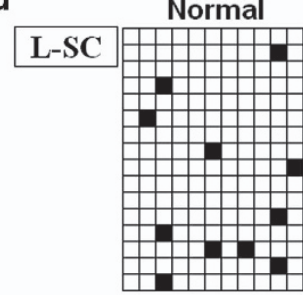

Normal

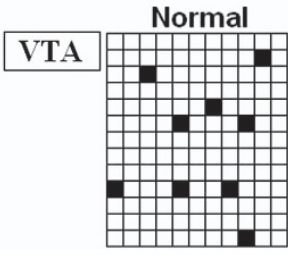

$\mathrm{CCl}$

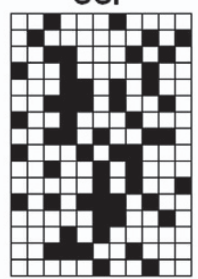

$\mathrm{CCl}$

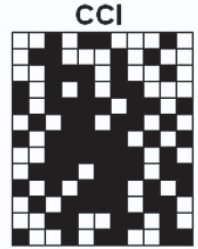

CCl+ISO-1

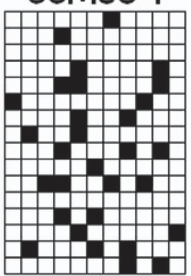

e
$\mathrm{CCl}+\mathrm{ISO}-1$

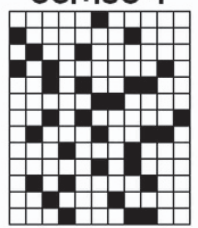

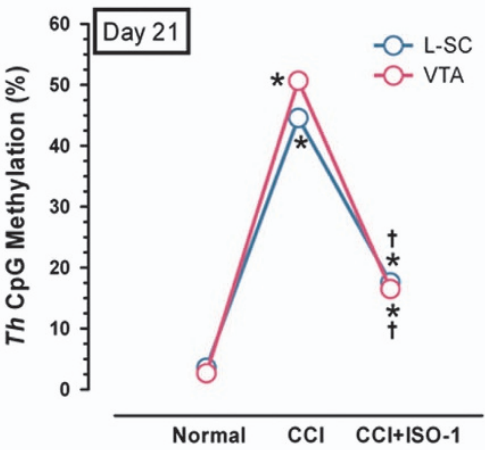

Figure 6 Th CpG islands methylation with or without ISO-1 following CCI. Eleven CpG islands within Th gene promoter and their corresponding locations are shown in a. In DRG, L-SC, VTA, NAc and PFc, BiSS results of these CpG islands' methylation and the methylation percentage in normal and $\mathrm{CCl}$ mice on day 21 post $\mathrm{CCl}$ are shown $(\mathbf{b}, \mathbf{c}),{ }^{* *} P<0.01$ vs normal mice. Data are shown as the means \pm s.e.m.'s and were analyzed with Student's $t$-test. Then, ISO-1 was i.t. or i.c.v. injected for 2 weeks from day 7 post nerve injury, and Th gene methylation in L-SC/VTA in normal, $\mathrm{CCl}$ and $\mathrm{CCl}+\mathrm{ISO}-1$ mice was detected with BiSS (d, e), ${ }^{*} P<0.05$ vs normal mice; ${ }^{\dagger} P<0.05$ vs $\mathrm{CCl}$ mice. Data are shown as the means \pm s.e.m.'s and were analyzed with one-way ANOVA. Each grid row represents one Th promoter $\mathrm{CpG}$ island, and each column denotes one clone number in $\mathbf{b}$ and $\mathbf{d}$. White and black grid indicates unmethylated and methylated cytosine, respectively. $\mathrm{N} 1=9$ (clone number in b); $\mathrm{n} 2=16$ (L-SC clone number in d); $\mathrm{n} 3=13$ (VTA clone number in d). BiSS, bisulfite sequencing; CCI, chronic constriction injury; DRG, dorsal root ganglion; L-SC, lumbar spinal cord L5-6; NAc, nucleus accumbens; PFc, prefrontal cortex; VTA, ventral tegmental area..

biologically strongly associated forms of epigenetic regulation. Accordingly, we are curious about the possible histones involved in MIF-associated Th gene methylation regulation. Our previous work suggested that lysine methyltransferases G9a and G9a-like protein (Glp) partially control dopaminergic transmission by methylating Th $\mathrm{CpG}$ sites in the VTA and contributing to pain hypersensitivity in spared nerve injuryinduced neuropathic pain ${ }^{23}$ because two related mammalian lysine methyltransferases, G9a/Glp heteromeric complex, could directly induce DNA methylation or indirectly induce $\mathrm{H} 3 \mathrm{~K} 9$ to finally silence target gene transcription. ${ }^{46}$ Additionally, evidence has shown the essential role of G9a repression and its reduction of $\mathrm{H} 3 \mathrm{~K} 9 \mathrm{me} 2$ in cocaine-induced structural and behavioral plasticity, ${ }^{47}$ while cocaine's effects depend on reuptake inhibition of presynaptic dopamine. ${ }^{48}$ Collectively, it is strongly speculated that there is involvement of G9a-related H3K9 regulation in MIF-associated Th gene hypermethylation. In addition, H3K9-specific HMTase Suv39H1 and its recruitment of heterochromatin protein 1 (HP1) were found to mediate DNA methylation-dependent histone modification and epigenetic transcription repression. ${ }^{49}$ Therefore, we tentatively detected G9a, SUV39H1, HP1, H3K9me2 and H3K9me3 expression in L-SC and VTA in our study and explored their possible roles in MIF-associated $T h$ gene methylation regulation. 


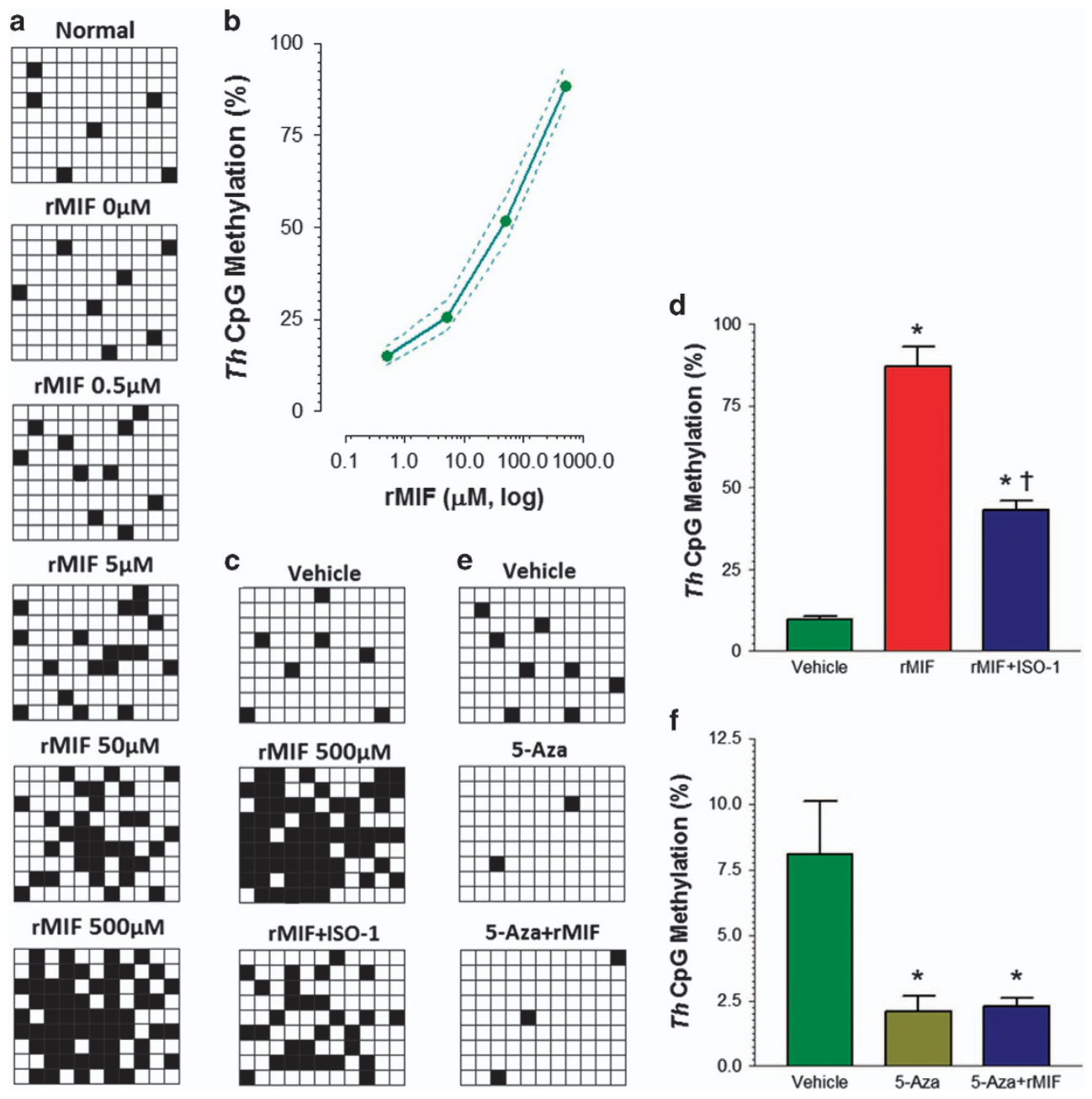

Figure 7 Relationship of migration inhibitory factor (MIF) and Th CpG island methylation in cultured dopaminergic neurons. Ascending doses of rMIF $(0,0.5,5,50$ and $500 \mu \mathrm{m})$ were applied to cultured dopaminergic neurons for 30 min at $37^{\circ} \mathrm{C}$, Th promoter $\mathrm{CpG}$ island methylation level was detected with BiSS $(\mathbf{a}, \mathbf{b})$. When ISO-1 was co-incubated, Th promoter CpG island methylation level in vehicle, rMIF, and $\mathrm{rMIF}+\mathrm{ISO}-1$ dopaminergic neurons was observed with BiSS as well (c, d), ${ }^{*} P<0.05$ vs vehicle; ${ }^{\dagger} P<0.05$ vs $\mathrm{rMIF}$ ). In addition, the Th methylation level was also evaluated in vehicle, 5-Aza and 5-Aza+rMIF incubation dopaminergic neurons (e, f), ${ }^{*} P<0.05$ vs vehicle). Each grid row represents one Th promoter $\mathrm{CpG}$ island, and each column denotes one clone number in a, c and e. White and black grid indicates unmethylated and methylated cytosine, respectively. Data are shown as the means \pm s.e.m.'s and were analyzed with one-way ANOVA. $N=9$ (clone number in $\mathbf{a}, \mathbf{c}$ and $\mathbf{e}$ ).

Western blot results showed that G9a, SUV39H1, H3K9me2 and $\mathrm{H} 3 \mathrm{~K} 9 \mathrm{me} 3$ expression increased on day 14 following CCI either in L-SC or in VTA (Figure 8a and b). Either i.t. or i.c.v. MIF-inhibitor ISO-1 injection with an ascending dose of $0,1.0$, 10.0 and $100.0 \mu \mathrm{g}$ on day 7 post injury for 2 weeks could block CCI-induced G9a, SUV39H1, H3K9me2 and H3K9me3 upregulation in L-SC and VTA with a dose-dependent increasing tendency $(100.0>10.0>1.0>0 \mu \mathrm{M}$ ) (Figure $8 \mathrm{c}$ and d). Further, $\mathrm{CHiP}$ assay was performed to provide a direct snapshot of G9a, SUV39H1 and HP1 recruitment, as well as $\mathrm{H} 3 \mathrm{~K} 9 \mathrm{me} 2$ and H3K9me3 enrichment, in Th promoter regions. Consistent with western blot results, increased recruitment of G9a and SUV39H1, but not HP1, as well as increased enrichment of H3K9me2 and H3K9me3 following CCI in both L-SC and VTA was observed, and this increase could be significantly alleviated following i.t. or i.c.v. injection of ISO-1 $30 \mu \mathrm{g}$ per day for 2 weeks from day 7 post nerve injury (Figure $8 \mathrm{e}-\mathrm{i}$ ). Thus, we strongly that suspect nerve injury-induced HMTase G9a and SUV39H1 upregulation, as well as the resulting $\mathrm{H} 3 \mathrm{~K} 9 \mathrm{me} 2$ and $\mathrm{H} 3 \mathrm{~K} 9 \mathrm{me}$, underlies Th gene methylation following CCI, and MIF might be the upstream regulator of this cascade.

\section{Relationship of MIF to HMTase G9a/SUV39H1 and H3K9 methylation}

Based on the above results, we propose the following scientific hypothesis: acting as an upstream regulator, MIF in L-SC and VTA induced HMTase G9a/SUV39H1 recruitment and H3K9 methylation alteration and finally potentiated high Th gene methylation. To verify our speculation, different inhibitors or activators were applied in primary cultured dopaminergic neurons to intervene in this regulation pathway and to observe G9a/SUV39H1, H3K9me2/H3K9me3 expression, as well as the 
final Th gene promoter CpG islands methylation profile. influence on rMIF $(500 \mu \mathrm{M})$-induced G9a/SUV39H1 and Western blot analysis showed that preincubation of the DNA H3K9me2/H3K9me3 upregulation (Figure 9a). The histone demethylation agent 5 -Aza $(15 \mu \mathrm{M})$ at $37^{\circ} \mathrm{C}$ for $30 \mathrm{~min}$ had no deacetylase inhibitor TSA (5 $\left.\mu \mathrm{M}\right)$ decreased rMIF induced

a

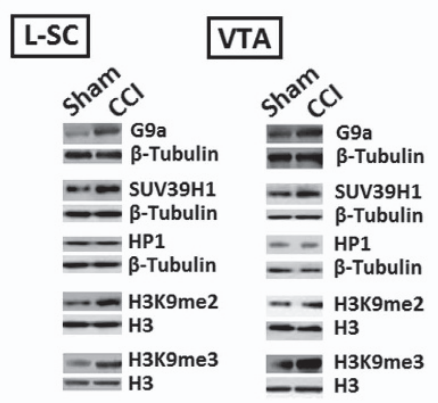

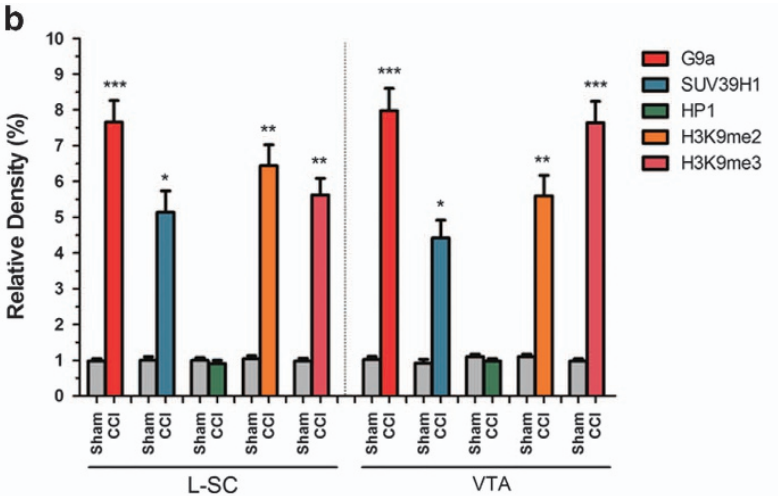

C

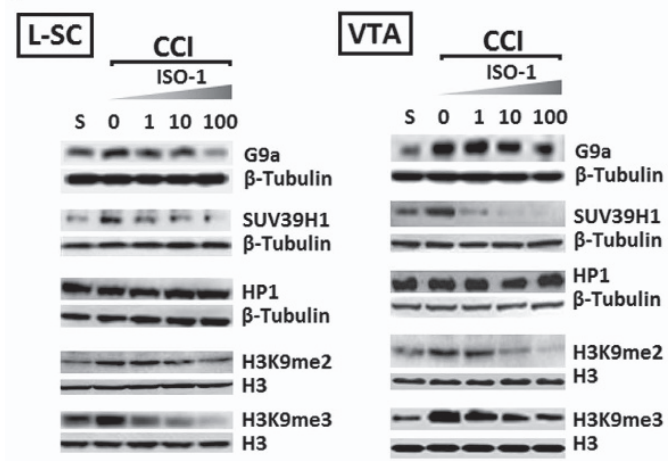

d

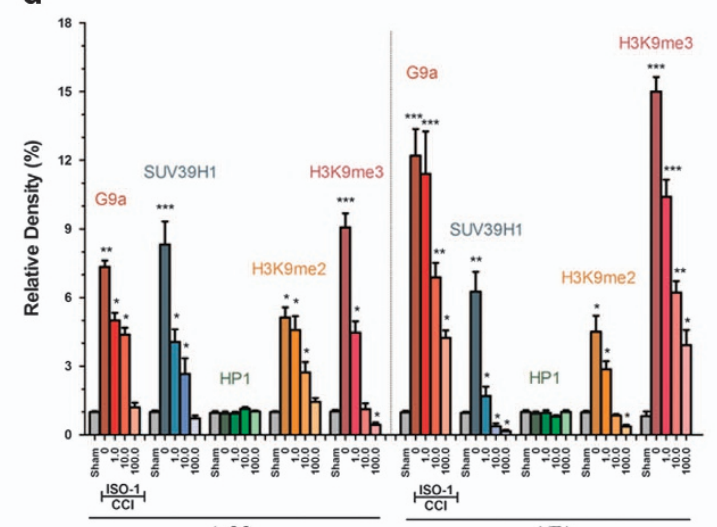

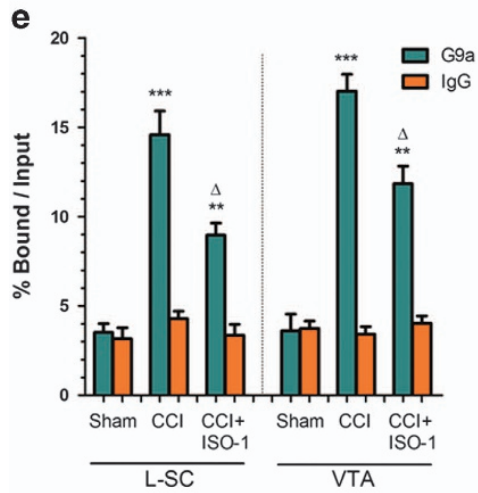
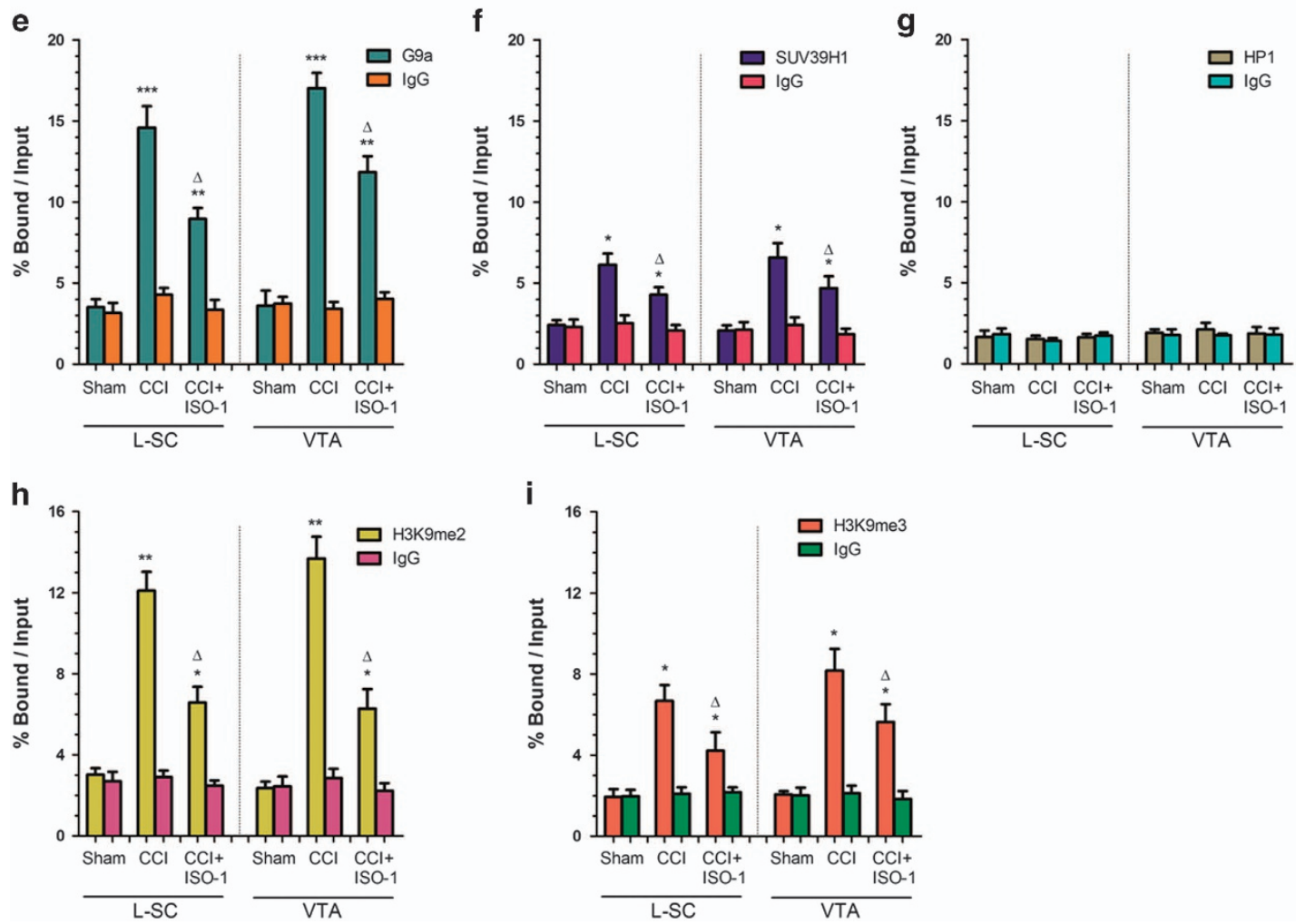
H3K9me2/H3K9me3 upregulation but not G9a/SUV39H1 high expression (Figure $9 \mathrm{~b}$ ). In contrast to TSA, histone deacetylase HDAC1 (30 $\mu \mathrm{M})$ further increased rMIF-induced H3K9me2/ H3K9me3 upregulation but not high G9a/SUV39H1 expression (Figure 9c). In addition, the G9a inhibitor BIX (15 $\mu \mathrm{M})$ blocked rMIF-induced G9a and $\mathrm{H} 3 \mathrm{~K} 9 \mathrm{me} 2 / \mathrm{H} 3 \mathrm{~K} 9 \mathrm{me} 3$ upregulation; however, it had no effect on SUV39H1 expression (Figure 9d). Further, the SUV39H1 inhibitor chaetocin (100 nM) blocked rMIF-induced SUV39H1 and H3K9me2/ H3K9me3 upregulation; however, it had no effect on G9a expression (Figure 9e), while no expression change in HP1 following rMIF, with or without various regulators, was observed. BiSS results suggested that Aza, TSA, BIX, Chaetocin reversed rMIF-induced Th $\mathrm{CpG}$ methylation in dopaminergic neurons, while HDAC1 increased rMIF-induced Th $\mathrm{CpG}$ methylation percentages (Figure 9f).

All of these results suggest that MIF functioned as an upstream regulator by mediating HMTase G9a/SUV39H1 upregulation and then methylating downstream $\mathrm{H} 3 \mathrm{~K}$, resulting in high dopaminergic neuron $T h$ gene $\mathrm{CpG}$ site methylation. Additionally, a report showed that G9a is interdependent with other HMTase, such as SUV39H1 and ESET, due to the same substrate specificity, ${ }^{50}$ so the contributing role of G9a and SUV39H1 in mediating H3K9 methylation was further explored. G9a/SUV39H1/HP1 recruitment to, as well as $\mathrm{H} 3 \mathrm{~K} 9 \mathrm{me} 2 / \mathrm{H} 3 \mathrm{~K} 9 \mathrm{me} 3$ enrichment in, the Th promoter was assayed with ChiP following rMIF $500 \mu \mathrm{M}$ incubation for $30 \mathrm{~min}$ at $37^{\circ} \mathrm{C}$. The ChiP results showed an increase in G9a/SUV39H1 but not HP1 occupancy, as well as an increase in $\mathrm{H} 3 \mathrm{~K} 9 \mathrm{me} 2 / \mathrm{H} 3 \mathrm{~K} 9 \mathrm{me} 3$ enrichment following rMIF in cultured dopaminergic neurons (Figure $9 \mathrm{~g}$ and $\mathrm{h}$ ). Further, after incubation for $30 \mathrm{~min}$ at $37^{\circ} \mathrm{C}$ with recombinant G9a $(5 \mu \mathrm{M})$ or SUV39H1 $(10 \mu \mathrm{M})$, ChiP assay indicated that either G9a or SUV39H1 could increase $\mathrm{H} 3 \mathrm{~K} 9 \mathrm{me} 2$ and $\mathrm{H} 3 \mathrm{~K} 9 \mathrm{me} 3$ expression, while a combination of G9a and SUV39H1 application produced an even more prominent increase (Figure 9i), suggesting an independent and cooperative relationship between G9a and SUV39H1 in potentiating downstream H3K9 methylation.
Pain behaviors following different inhibitors or activators According to our scientific hypothesis, the Th promoter CpG methylation level underlies the final pain phenotype. We thus respectively measured allodynia, mechanical and thermal hyperalgesia in CCI mice after administering different inhibitors and activators of this regulatory pathway. Pain behavior results showed that i.t. ISO-1 $30 \mu \mathrm{g}$ per day treatment for 2 weeks from day 7 post CCI significantly improved the decreased withdrawal threshold in CCI mice. Further, ISO-1 had synergic effects on nociception improvement when additionally administered with TSA ( $25 \mu \mathrm{g}$ per day), BIX (30 $\mu \mathrm{g}$ per day), and 5-Aza (10 $\mu \mathrm{g}$ per day) but not with HDACs (50 $\mu \mathrm{g}$ per day) and DAT-Sap (10 $\mu \mathrm{g}$ per day) (Figure 10a and b). Similar to i.t. treatment, i.c.v. injection of ISO+TSA/ BIX/Aza synergistically improved CCI-induced allodynia more than ISO alone, while ISO+HDACs/DAT-Sap mice had comparable withdrawal thresholds to CCI mice (Figure 10c and d).

Consistent with allodynia, the mechanical and heat hyperalgesia results also showed that either i.t. or i.c.v. treatment of a combination of ISO with TSA, BIX or Aza significantly improved, while ISO alone to a lesser extent improved, hyperalgesia compared to CCI mice; however, ISO with HDACs or DAT-Sap showed no significant difference in CCI mice with hyperalgesia (Figure 10e-h).

These results suggested that different inhibitors or activators alone or in combination induced pain phenotypes consistent with their respective Th promoter CpG island methylation levels shown in Figure 9f, robustly verifying our scientific hypothesis depicted in Figure 11a. Nerve injury-induced MIF expression in L-SC and VTA, activated HMTase G9a and SUV39H1, potentiated H3K9 methylation, enhanced Th gene CpG sites methylation, inhibited TH transcription, decreased dopamine content, and as a result decreased dopaminergic descending inhibition, contributing to pain hypersensitivity. Different regulators and their targets, as well as theoretical regulation pathways in this study, are shown in Figure 11b. Regulators including ISO-1, BIX01294, Chaetocin, TSA and 5Aza showed inhibitory effects on the pathway and ultimately improved hyperalgesia, while red arrows, mainly HDACs

Figure 8 G9a, SUV39H1, HP1, H3K9me2 and H3K9me3 expression in L-SC and VTA. G9a, SUV39H1, HP1, H3K9me2 and H3K9me3 expression was measured with western blot analysis in sham and chronic constriction injury (CCl) mice. Representative western blot gel images and their relative density in L-SC and VTA on day 14 post injury are presented in $\mathbf{a}$ and $\mathbf{b}\left({ }^{*} P<0.05,{ }^{* *} P<0.01,{ }^{* * *} P<0.001\right.$ vs sham mice. Data are shown as the means \pm s.e.m.'s and were analyzed with Student's $t$-test). Then, the migration inhibitory factor (MIF) inhibitor ISO-1, at ascending doses of $0,1.0,10.0$ and $100.0 \mu \mathrm{M}$, was i.t. or i.c.v. injected on day 7 post injury for 2 weeks. G9a, SUV39H1, HP1, H3K9me2 and H3K9me3 expression in L-SC and VTA on day 21 post nerve injury was further evaluated, and representative western blot gel images and their relative densities are presented in $\mathbf{c}$ and $\mathbf{d}\left({ }^{*} P<0.05,{ }^{*} P<0.01,{ }^{* *} P<0.001\right.$ vs sham mice. Data are shown as the means \pm s.e.m.'s and were analyzed with one-way ANOVA). Recruitment levels of G9a, SUV39H1, HP1, H3K9me2 and H3K9me3 in the Th promoter region in L-SC and VTA of sham, CCl and CCl+ISO-1 mice, measured with CHiP and normalized by input, are shown in $\mathbf{e}-\mathbf{i}\left({ }^{*} P<0.05\right.$ vs sham mice, $\triangle P<0.05$ vs $\mathrm{CCl}$ mice. Data are shown as the means \pm s.e.m.'s and were analyzed with one-way ANOVA). $\beta$-tubulin or H3 was used as a loading control for western blot, and lgG was used as a negative control in CHiP assay; $n=15$ in each group. 
and DAT-Saporin, further worsened neuropathic pain behaviors.

\section{DISCUSSION}

Herein, we demonstrate that L-SC and VTA are two major locations with significant upregulation of MIF and negatively related DA downregulation after CCI. Levels of both MIF and DA displayed time-dependent changes along with the behavior trajectory, and they were reversed by i.t. or i.c.v. MIF tautomerase inhibitor ISO-1. Further, reversal role by ISO-1 also had effects on $\mathrm{TH}$ expression and $\mathrm{Th}$ gene methylation in L-SC and VTA. Moreover, ISO-1 could reduce rMIF-induced methylation of the Th gene in cultured dopaminergic neurons, which was displayed in a dose-dependent, increasing manner. In addition, ISO-1 could decrease HMTase G9a/SUV39H1 enrichment, as well as $\mathrm{H} 3 \mathrm{~K} 9 \mathrm{me} 2 / \mathrm{H} 3 \mathrm{~K} 9 \mathrm{me} 3$ within the Th a
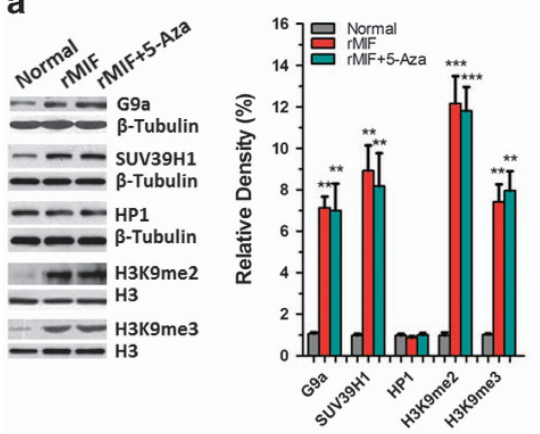

b

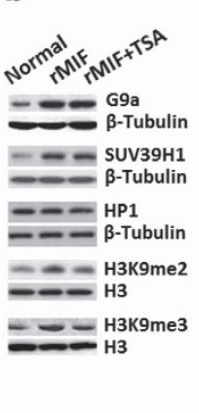

C
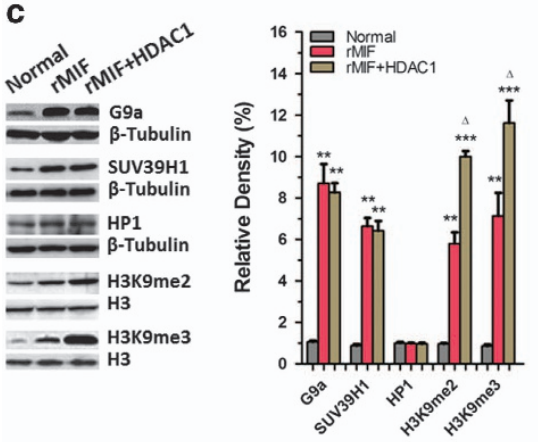

d

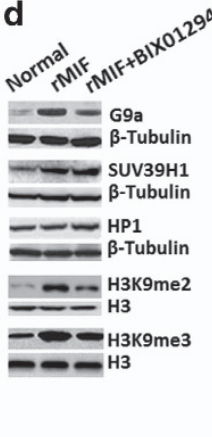

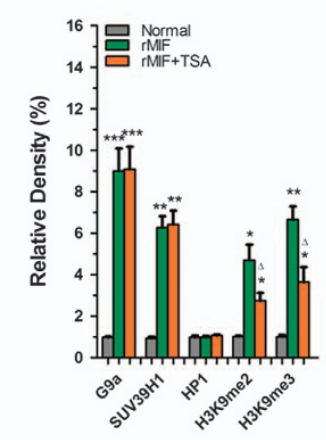
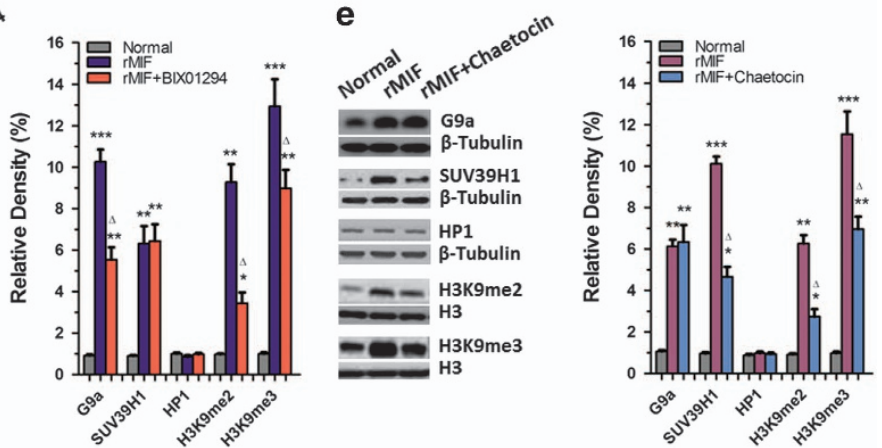

f
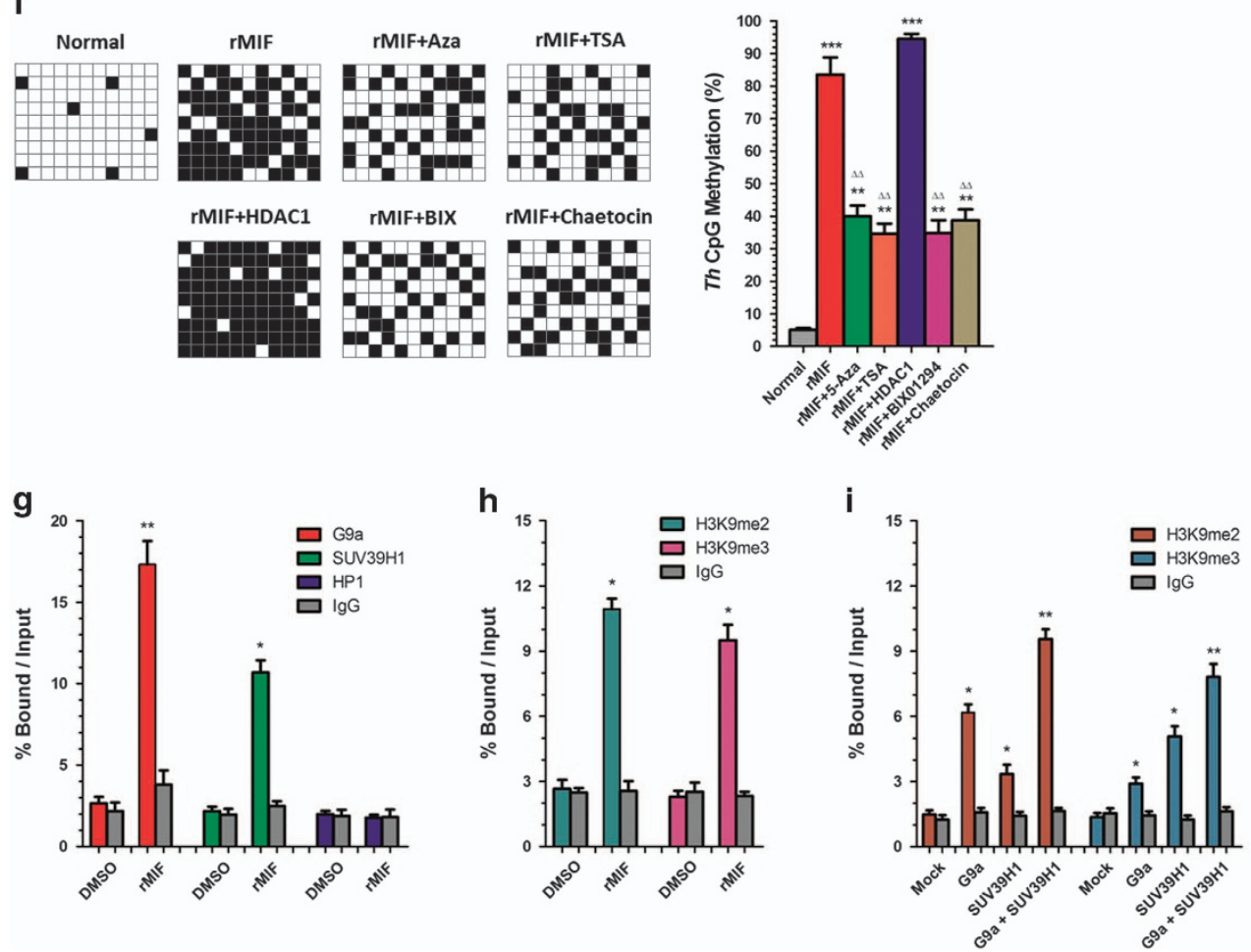
promoter region following CCI in L-SC and VTA. Finally, an independent and cooperative effect of G9a and SUV39H1 in inducing $\mathrm{H} 3 \mathrm{~K} 9 \mathrm{me} 2 / \mathrm{me} 3$ was demonstrated by regulators, including 5-Aza, TSA, HDAC1, BIX and chaetocin, in cultured dopaminergic neurons. Correspondingly, Th promoter $\mathrm{CpG}$ site methylation and pain behaviors also exhibited changes with the molecular changes following various regulators. These data emphasize that MIF functions as an inhibitory factor curbing dopaminergic descending inhibition of peripheral nerve injuryinduced hypersensitivity through mediating Th gene methylation by G9a/SUV39H1-associated H3K9me2/H3K9me3 methylation.

MIF, a $12.5-\mathrm{kDa}$ protein, was initially described as a proinflammatory cytokine, and it has recently been implicated in inflammatory and neuropathic pain. ${ }^{15,17,42}$ Although ubiquitously expressed throughout the central nervous system, our previous work and that of others mainly focused on MIF upregulation in the spinal cord following sciatic nerve chronic constriction injury or spinal compression injury. ${ }^{16,51}$ However, MIF expression in supraspinal regions in response to nerve injury was not evaluated previously, and this study revealed increased MIF expression in the VTA, which is a heterogeneous brain structure in which dopaminergic neurons play distinct roles, not only in rewards and aversive processing, associative learning, general motivational salience and cognition ${ }^{52}$ but also in pain regulation. ${ }^{23,53,54}$ Notably, MIF levels were upregulated from 2 to 4 days and peaked at nearly 14-21 days post CCI, and these characteristic changes were coincident with the pain behavior trajectory; further, either i.t. or i.c.v. MIF inhibitor ISO-1 could improve hypersensitivity, collectively indicating, on the one hand, that both spinal and supraspinal MIF contributed to pain perception and, on the other hand, as verified by others, ${ }^{15,16,51}$ that high or sustained MIF production might predict the duration and severity of the pathological and neurological outcomes underlying neuropathic pain.

L-SC and VTA are also two regions with dopamine downregulation. Dopaminergic neurons originate from VTA and project to striatum/NAc or prefrontal cortex to comprise the mesolimbic and mesocortical pathways, respectively. There are also dopaminergic projections to the spinal cord. Dopamine- containing fibers and terminals are widely distributed, while dopaminergic cell bodies are absent from the spinal dorsal horn. ${ }^{55}$ A previous study showed that dopaminergic neurons of the A11 diencephalospinal system are the only origin of dopaminergic projections to the spinal cord, and these neurons play neuromodulatory roles in nociceptive input processing. ${ }^{56,57}$ Direct VTA stimulation may decrease spinal dorsal horn neurons activity to exhibit an analgesic effect. ${ }^{54}$ Collectively, the decreased dopamine in the L-SC is largely attributed to decreased dopaminergic projection from VTA in our study. Compared to an enormous number of reports devoted to mesolimbic and mesocortical dopamine pathways in neuropathic pain, ${ }^{58,59}$ this VTA to L-SC descending dopaminergic pathway in pain regulation after peripheral nerve injury has received scant attention. This turnover role of ISO-1 on dopamine also affected $\mathrm{TH}$, but not DAT, expression, largely restoring CCI-induced TH downregulation in L-SC and VTA. Thus, the decrease in dopamine synthesis but not an increase in dopamine reuptake could lead to the reduction of dopamine levels in the L-SC and VTA after CCI. Even for the mesolimbic dopaminergic pathway, various results were observed with regard to dopamine and $\mathrm{TH}$, as well as DAT expression, following peripheral nerve injury. In the spared nerve injury (SNI) model of neuropathic pain, reduced dopamine levels resulted from upregulated DAT, but TH expression in the NAc was not observed; ${ }^{60}$ elevated extracellular dopamine levels and reduced TH expression in the NAc were also reported after SNI. ${ }^{61}$ In contrast, increased TH and dopamine levels were demonstrated after unilateral CCI of the sciatic nerve. ${ }^{62}$ Such a discrepancy might depend on different animal models, distinct cerebrospinal regions, and the observation time points, increasing our knowledge of the neuroplasticity of dopaminergic pathways in pain regulation.

A salient finding of this study is MIF region-specific targeted DNA methylation of Th promoter $\mathrm{CpG}$ islands contributing to dopamine downregulation in the L-SC and VTA. DNA methylation is best known as an epigenetic regulator of postmitotic neurons to repress gene transcription without alteration of the original DNA sequences. ${ }^{63,64}$ Although algogenic cytokine MIF per se is necessary and sufficient to sensitize

Figure 9 Relationship of HMTase G9a/SUV39H1 and H3K9 methylation. Cultured dopaminergic neurons on day 7 in vitro were treated with rMIF in combination with the DNA demethylation agent Aza, histone deacetylase inhibitor TSA, histone deacetylase HDAC1, G9a inhibitor BIX and SUV39H1 inhibitor chaetocin for 30 min at $37^{\circ} \mathrm{C}$. G9a, SUV39H1, HP1 and H3K9me2/H3K9me3 expression, as well as Th promoter $\mathrm{CpG}$ island methylation profiles, was evaluated with western blot and BiSS. Representative western blot gel images and the relative density of G9a, SUV39H1, HP1 and H3K9me2/H3K9me3 expression are shown in a-e after Aza, TSA, HDAC1, BIX and chaetocin treatment, respectively ( ${ }^{* *} P<0.05,{ }^{* *} P<0.01$ vs normal; $\triangle P<0.05$ vs rMIF). BiSS results of $T h$ CpG methylation profiles, as well as their methylation percentages in normal, rMIF, rMIF+Aza, rMIF+TSA, rMIF+HDAC1, rMIF+BIX and rMIF+chaetocin neurons, are shown in $\mathbf{f}$ ${ }^{* *} P<0.05,{ }^{* *} P<0.01$ vs normal; $\triangle \triangle P<0.05$ vs rMIF. Each grid row represents one Th promoter CpG island, and each column denotes one clone number. White and black grid indicates unmethylated and methylated cytosine, respectively. $N=9$ in clone number. G9a, SUV39H1, HP1 and H3K9me2/H3K9me3 levels following rMIF in dopaminergic neurons were measured with ChiP and are shown as bound/input percentages in $\mathbf{g}$ and $\mathbf{h}\left({ }^{*} P<0.05,{ }^{*} P<0.01\right.$ vs DMSO). Recruitment levels of H3K9me2 and H3K9me3 following G9a and SUV39H1 application alone or in combination in dopaminergic neurons were measured and are shown in $\mathbf{i}\left({ }^{*} P<0.05\right.$, ${ }^{* *} P<0.01$ vs Mock). ChiP results are the average of three independent experiments. Data are shown as the means \pm s.e.m.'s and were analyzed with one-way ANOVA. BiSS, bisulfite sequencing; MIF, migration inhibitory factor. 
nociceptive neurons, their possible roles in Th gene DNA methylation and transcriptional silencing of $\mathrm{TH}$ expression in neuropathic pain have not been determined previously. As proved, consistent with the induction of MIF expression in L-SC and VTA, increased Th gene promoter CpG site methylation was observed as well. Further, rMIF dosedependently increased $T h$ methylation in cultured dopaminergic neurons, and the turnover effect of MIF inhibitor ISO-1 for TH also applied for high Th high both in vivo and in vitro. Furthermore, the pain improvement efficacy of ISO-1 was paralleled with corresponding Th CpG site methylation status. These data provided functional evidence for the essential role of MIF-mediated somatosensory system maladaptation of VTA to the L-SC descending dopa-

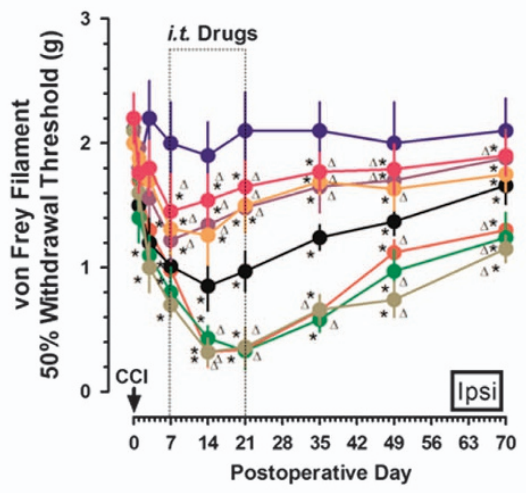

C

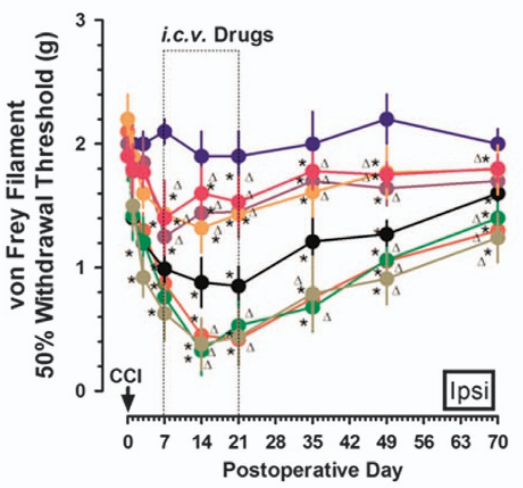

b

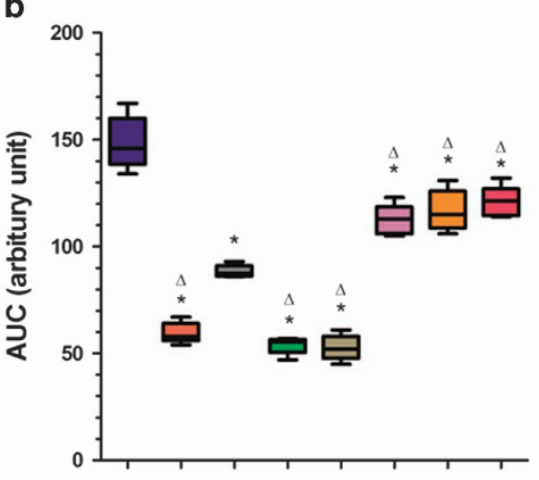

d

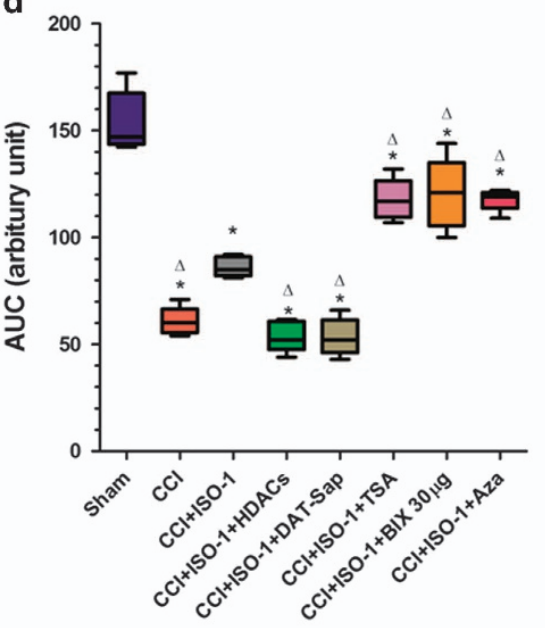

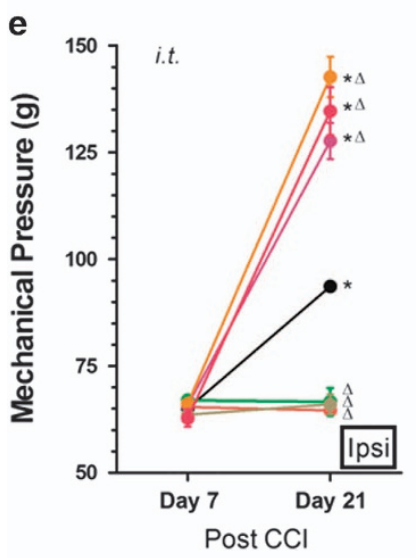
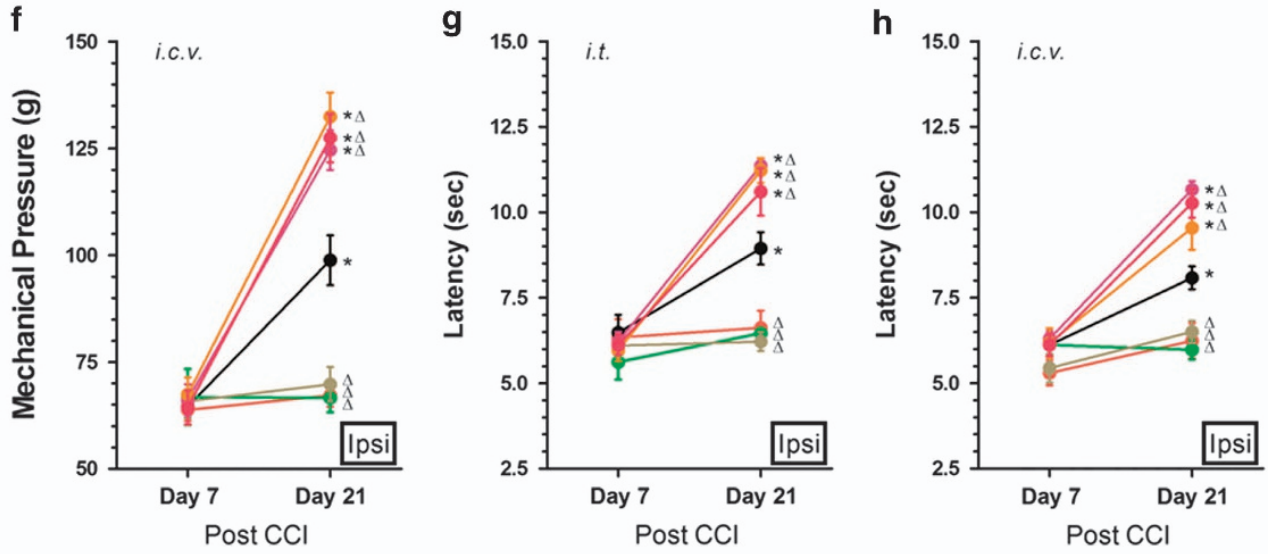

$\rightarrow \mathrm{CCl}$

- $\mathrm{CCl}+\mathrm{ISO}-1 \quad \rightarrow \mathrm{CCl}+\mathrm{ISO}-1+\mathrm{TSA}$

$\because \mathrm{CCl}+\mathrm{ISO}-1+\mathrm{HDACs} \rightarrow \mathrm{CCl}+\mathrm{ISO}-1+\mathrm{BIX} 30 \mu \mathrm{g}$

$\rightarrow \mathrm{CCl}+\mathrm{ISO}-1+$ DAT-Sap $\rightarrow \mathrm{CCl}+\mathrm{ISO}-1+\mathrm{Aza}$ 
minergic pathway in neuropathic pain.

We also provide novel evidence for the involvement of G9a in nerve injury-induced neuropathic pain. As histone methyltransferases, G9a and G9a-like protein (Glp) often exist as a G9a/Glp heteromeric complex to biochemically catalyze mono-, di-, and trimethylation reactions on $\mathrm{H} 3 \mathrm{~K} 9 .{ }^{65}$ Functionally, G9a/Glp can directly affect DNA methylation or interact with $\mathrm{H} 3 \mathrm{~K} 9$ methylation to indirectly control DNA methylation and silence target gene transcription. ${ }^{46}$ Several recent studies have suggested the participation of G9a in pain regulation. For example, nerve injury consistently increased G9a and H3K9me2 occupancy at the mu-opioid receptor (MOR) gene promoter in DRG and induced a long-term reduction in the expression level of MORs, decreasing opioid analgesic efficacy during neuropathic pain treatment; ${ }^{66,67}$ it was also reported that nerve injury increased G9a activity in the DRG, epigenetically silencing $\mathrm{K}^{+}$channel genes to promote chronic pain development after nerve injury. ${ }^{68,69}$ In the present study, we particularly found increased G9a, $\mathrm{H} 3 \mathrm{~K} 9 \mathrm{me} 2$ and $\mathrm{H} 3 \mathrm{~K} 9 \mathrm{me} 3$ in the L-SC and VTA. Interestingly, in Laumet's study, upregulated G9a was only observed in the DRG but not in the L-SC after SNL, with supraspinal regions not detected, ${ }^{68}$ and in our previous work, G9a was detected in cerebrospinal regions, including DRG, L-SC, VTA, NAc and PFc, after SNI; however, increased G9a was observed only in the VTA. ${ }^{23}$ The underlying reason for this difference might be the type of peripheral nerve injury, although CCI, SNL and SNI are well established neuropathic pain models, although they have distinctive molecular, cellular and epigenetic characteristics. ${ }^{70}$ Increased global DNA methylation and $\mathrm{MeCP} 2$ expression in the lumbar spinal cord 14 days following CCI were previously proved $;{ }^{71}$ in contrast, SNI resulted in downregulation of MeCP2, DNMTs and a subset of HDACs in the superficial dorsal horn, ${ }^{72}$ and combined with our results of increased G9a in the VTA and L-SC, this result underscored the mechanistic differences underlying distinct pain models. ${ }^{73}$ In addition to G9a, H3K9-specific HMTase SUV39H1 was also found to participate in $\mathrm{H} 3 \mathrm{~K} 9 \mathrm{me} 2$ and $\mathrm{H} 3 \mathrm{~K} 9 \mathrm{me} 3$ following CCI in L-SC and VTA. One recent work showed that SUV39H1 contributed to nerve injury-induced hyperalgesia by controlling MOR expression in the injured DRG following SNI ${ }^{74}$ however, the underlying epigenetic mechanism was not explored. Our study for the first time demonstrated the contributing role of SUV39H1 and its induction of $\mathrm{H} 3 \mathrm{~K} 9 \mathrm{me} / \mathrm{H} 3 \mathrm{~K} 9 \mathrm{me} 3$ in dopaminergic epigenetic inhibition post CCI.

In addition to the increased expression of G9a, SUV39H1 and $\mathrm{H} 3 \mathrm{~K} 9 \mathrm{me} 2 \mathrm{H} 3 \mathrm{~K} 9 \mathrm{me} 3$ in the L-SC and VTA, more importantly, we determined the relationship of MIF, G9a, SUV39H1, H3K9me2/H3K9me3, Th gene methylation, and their relative contributions to pain hypersensitivity after CCI. As shown by western blot analysis and ChiP, ISO-1 inhibited G9a, SUV39H1, and H3K9me2/H3K9me3 expression in a dosedependent, increasing manner, demonstrating that MIF acted as an upstream regulator of G9a, SUV39H1, and H3K9me2/ H3K9me3. Moreover, in primary cultured dopaminergic neurons, G9a inhibition by BIX or SUV39H1 inhibition by chaetocin downregulated $\mathrm{H} 3 \mathrm{~K} 9$ methylation, while conversely, H3K9 inhibition by TSA had no effect on G9a and SUV39H1 expression, collectively indicating that G9a or SUV39H1induced $T h$ gene $\mathrm{CpG}$ methylation was $\mathrm{H} 3 \mathrm{~K} 9 \mathrm{me} 2 / \mathrm{H} 3 \mathrm{~K} 9 \mathrm{me} 3$ dependent. An independent and cooperative relationship between G9a and SUV39H1 in potentiating downstream H3K9 methylation was also proved by ChiP assay in vitro. In addition, BiSS results showed that Aza/TSA/BIX/chaetocin were largely suppressed, while HDAC1 elevated rMIF-induced $T h$ gene CpG methylation in vitro, further verifying the "MIF-G9a/ SUV39H1-H3K9me2/H3K9me3-Th gene methylation pathway.

Finally, consistent with the molecular changes, combined treatments of ISO-1 with BIX 01294, TSA or 5-Aza, but not with HDACs and DAT-Saporin, produced a significantly greater pain improvement effect than ISO-1 alone, suggesting a cooperative interaction between MIF and G9a/SUV39H1$\mathrm{H} 3 \mathrm{~K} 9 \mathrm{me} 2 / \mathrm{H} 3 \mathrm{~K} 9 \mathrm{me} 3-\mathrm{Th}$ gene $\mathrm{CpG}$ methylation in the control of nerve injury-induced dysfunction of VTA to the L-SC dopaminergic pathway. Nevertheless, because multiple regulators on this pathway per se have pain regulation properties, it is difficult to determine the extent to which the analgesic effect of, e.g., ISO plus BIX, is attributable to their rescuing effects on decreased dopaminergic inhibitory effects. Other than the aforementioned potent antinociceptive effect of MIF inhibition via blocking effects, including sensitizing nociceptive neurons, promoting algogenic genes transcription, and activating central

Figure 10 Pain behaviors following different inhibitors or activators. Different inhibitors or activators of this pain regulatory pathway, including the migration inhibitory factor (MIF) inhibitor ISO-1, histone deacetylase HDACs, DAT inactivating protein DAT-Saporin, histone deacetylase inhibitor TSA, G9a inhibitor BIX and direct DNA demethylation agent Aza, were i.t. or i.c.v. injected with ISO-1 alone or ISO-1 combined with one of the other regulators on day 7 post nerve injury for 14 days, and pain behaviors, including $50 \%$ withdrawal threshold, mechanical pressure and thermal withdrawal latency, were observed throughout the 70 days post nerve injury. The ipsilateral $50 \%$ withdrawal threshold and corresponding AUC are shown in $\mathbf{a}$ and $\mathbf{b}$ following i.t. and in $\mathbf{c}$ and $\mathbf{d}$ following i.c.v. treatment. $\left({ }^{*} P<0.05\right.$ vs sham; $\Delta P<0.05$ vs CCI. Data are shown as the means \pm s.e.m.'s and were analyzed with two-way ANOVA.) In $\mathrm{CCl}, \mathrm{CCl}+\mathrm{ISO}-1, \mathrm{CCl}+\mathrm{ISO}-1$ +HDACs, $\mathrm{CCl}+\mathrm{ISO}-1+\mathrm{DAT}-\mathrm{Sap}, \mathrm{CCl}+\mathrm{ISO}-1+\mathrm{TSA}, \mathrm{CCl}+\mathrm{ISO}+\mathrm{BIX}$ and $\mathrm{CCl}+\mathrm{ISO}-1+\mathrm{Aza}$ mice, mechanical pressure on days 7 and 21 is shown in $\mathbf{e}$ and $\mathbf{f}$ following i.t. and i.c.v. treatment, and thermal withdrawal latency is shown in $\mathbf{g}$ and $\mathbf{h}$ following i.t. and i.c.v. treatment $\left({ }^{*} P<0.05\right.$ vs day $7 ;{ }^{\Delta} P<0.05$ vs day 21 in $\mathrm{CCl}$ mice. Data are shown as the means \pm s.e.m.'s and were analyzed with Student's $t$-test for intra-group and two-way ANOVA for inter-group differences. $N=15$ in each group. $\mathrm{CCl}$, chronic constriction injury; DAT, dopamine transporter; HDAC, histone deacetylases. 

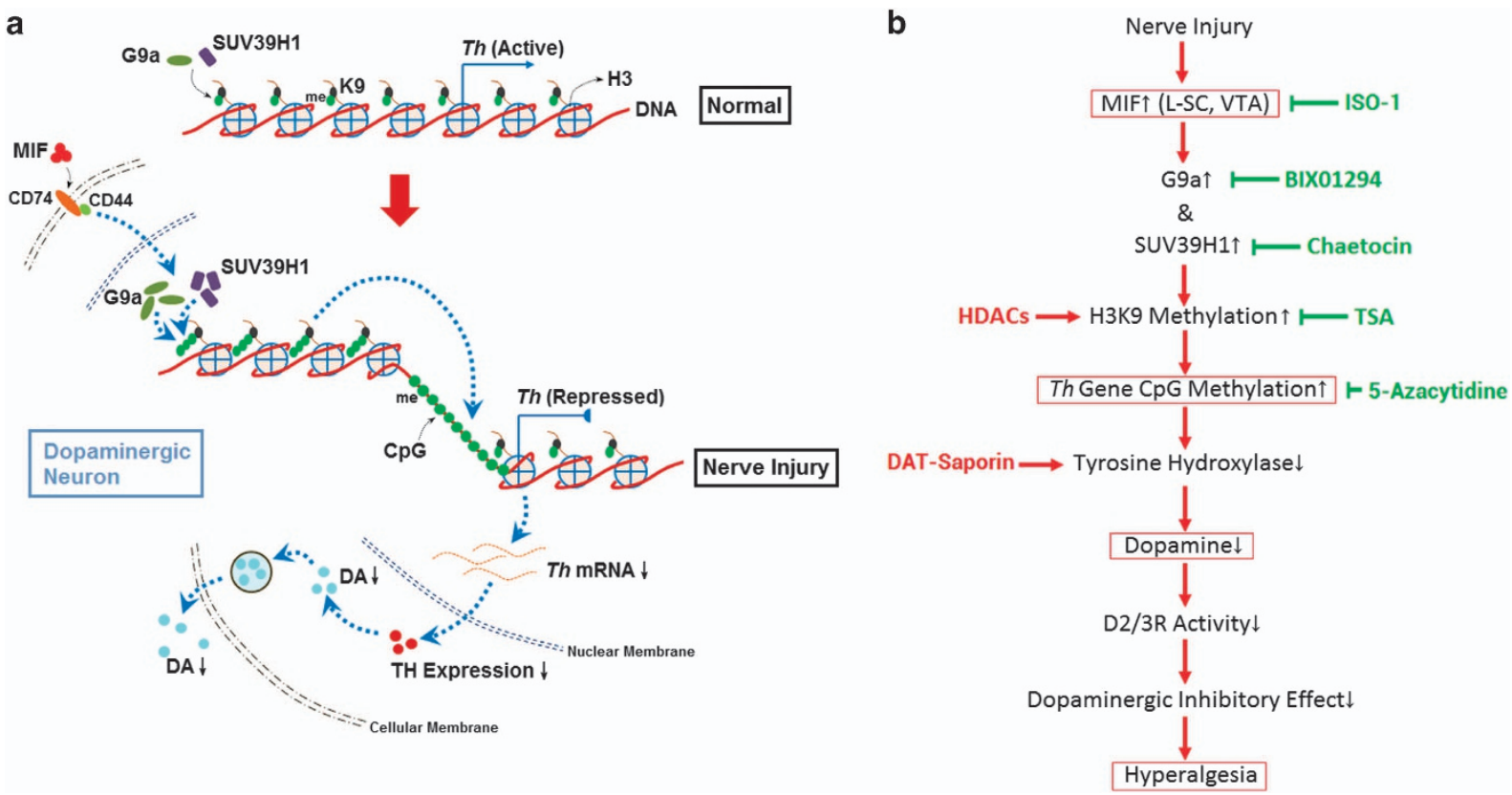

Figure 11 Schematic for migration inhibitory factor (MIF)-associated dopaminergic inhibition. Schematic shows the underlying changes in MIF-associated dopaminergic inhibition due to peripheral nerve injury (a). Upregulated MIF promotes recruitment of both G9a and SUV39H1 to methylate H3K9 be H3K9me2 and H3K9me3, enhancing Th promoter CpG islands being methylated, and then Th transcription decreases, consequently resulting in a reduction in dopamine (DA) production. Our study process is depicted in $\mathbf{b}$; the blue horizontal line shows an inhibitory effect for the pathway and ultimately improves the final hyperalgesia, while the red arrows denote a stimulatory effect for neuropathic pain behavior deterioration.

sensitization related signaling pathway, ${ }^{15,17}$ G9a inhibition also normalized the expression of nerve injury induction of many genes known to be involved in nociceptive regulations, such as calcium signaling, serotonin receptors, axonal guidance and G-protein-coupled receptor, ${ }^{66-69,75}$ restoration of the expression of which contributed to the analgesic effect of G9a inhibition. Regardless of these interpretations of the results, this study provided a new plausible mechanism underlying the analgesic effect of MIF-mediated descending dopaminergic pathway inhibition on neuropathic pain.

Before concluding, we should acknowledge several limitations of this study. Although previous work demonstrated that spinal neurons and microglia are major sources of MIF production after formalin-induced inflammatory and peripheral nerve injury-induced neuropathic hyperalgesia, ${ }^{16,42}$ the origins of MIF in L-SC and VTA following CCI were not detected in this study. Second, nerve injury-induced gene expression changes likely involve multiple epigenetic regulators, and it was also reported that G9a inhibition not only reduced H3K9me2 levels but also increased H3K9ac levels, ${ }^{76}$ and the coordinated network between methylation and acetylation at H3K9 could cooperatively induce target gene transcription inhibition, which was not tested herein. In fact, elucidating the integrated transcriptional crosstalk involved in altered gene expression in neuropathic pain remains a challenging task. Third, other than the MIF-related descending dopaminergic pathway in the L-SC and VTA, the roles of transient dopamine decrease in NAc and increased NE content in DRG, L-SC and
LC observed in our study have not been deeply explored. Therefore, further studies are warranted to more deeply clarify these points.

In conclusion, as shown in Figure 11, MIF also functions as a braking factor in curbing dopaminergic descending inhibition in peripheral nerve injury-induced hypersensitivity through mediation of Th gene methylation by G9a/SUV39H1-associated H3K9me2/H3K9me3 methylation. Our findings demonstrated that MIF-related dopaminergic descending inhibitory pathway dysfunction plays an essential role in the pathogenesis of nerve injury-induced neuropathic pain, suggesting that various interventions for these pathways might provide promising therapies for neuropathic pain.

\section{CONFLICT OF INTEREST}

The authors declare no conflict of interest.

\section{ACKNOWLEDGEMENTS}

This work was partially presented at the 2017 Annual Meeting of The American Society of Anesthesiologists (Boston, MA, USA) as a poster (A2240). This work was supported in part by the National Natural Scientific Foundation of China (NSFC, 81271242, 81371248 and 81600960), Qinghai Provincial Natural Scientific Foundation Innovation Group (2013-Z-921), Jiangsu Provincial Medical Youth Talent (QNRC2016103) and the Nanjing Medical Science Development Grant (YKK15162). 


\section{PUBLISHER'S NOTE}

Springer Nature remains neutral with regard to jurisdictional claims in published maps and institutional affiliations.

1 Colloca L, Ludman T, Bouhassira D, Baron R, Dickenson AH, Yarnitsky D et al. Neuropathic pain. Nat Rev Dis Primers 2017; 3: 17002.

2 Millan MJ. Descending control of pain. Prog Neurobiol 2002; 66: 355-474.

3 Wood PB. Role of central dopamine in pain and analgesia. Expert Rev Neurother 2008; 8: 781-797.

4 Fleetwood-Walker SM, Hope PJ, Mitchell R. Antinociceptive actions of descending dopaminergic tracts on cat and rat dorsal horn somatosensory neurones. J Physiol 1988; 399: 335-348.

5 Taniguchi W, Nakatsuka T, Miyazaki N, Yamada H, Takeda D, Fujita T et al. In vivo patch-clamp analysis of dopaminergic antinociceptive actions on substantia gelatinosa neurons in the spinal cord. Pain 2011; 152: 95-105.

6 Munro G. Dopamine $\mathrm{D}(1)$ and $\mathrm{D}(2)$ receptor agonism enhances antinociception mediated by the serotonin and noradrenaline reuptake inhibitor duloxetine in the rat formalin test. Eur J Pharmacol 2007; 575: 66-74.

7 Finnerup NB, Otto M, McQuay HJ, Jensen TS, Sindrup SH. Algorithm for neuropathic pain treatment: an evidence based proposal. Pain 2005; 118 : 289-305.

8 Bloom BR, Bennett B.. Mechanism of a reaction in vitro associated with delayed-type hypersensitivity. Science 1966; 153: 80-82.

9 Santos LL, Morand EF. Macrophage migration inhibitory factor: a key cytokine in RA, SLE and atherosclerosis. Clin Chim Acta 2009; 399: 1-7.

10 Sanchez-Zamora YI, Rodriguez-Sosa M. The role of MIF in type 1 and type 2 diabetes mellitus. J Diabetes Res 2014; 2014: 804519.

11 Kindt N, Journe F, Laurent G, Saussez S. Involvement of macrophage migration inhibitory factor in cancer and novel therapeutic targets. Oncol Lett 2016; 12: 2247-2253.

12 Kudrin A, Ray D. Cunning factor: macrophage migration inhibitory factor as a redox-regulated target. Immunol Cell Biol 2008; 86: 232-238.

13 Lerch JK, Puga DA, Bloom O, Popovich PG. Glucocorticoids and macrophage migration inhibitory factor (MIF) are neuroendocrine modulators of inflammation and neuropathic pain after spinal cord injury. Semin Immunol 2014; 26: 409-414.

14 Bacher M, Meinhardt A, Lan HY, Dhabhar FS, Mu W, Metz CN et al. MIF expression in the rat brain: implications for neuronal function. Mol Med 1998; 4: 217-230.

15 Alexander JK, Cox GM, Tian JB, Zha AM, Wei P, Kigerl KA et al. Macrophage migration inhibitory factor (MIF) is essential for inflammatory and neuropathic pain and enhances pain in response to stress. Exp Neurol 2012; 236: 351-362.

16 Wang F, Xu S, Shen X, Guo X, Peng Y, Yang J. Spinal macrophage migration inhibitory factor is a major contributor to rodent neuropathic painlike hypersensitivity. Anesthesiology 2011; 114: 643-659.

17 Wang F, Wu H, Xu S, Guo X, Yang J, Shen X. Macrophage migration inhibitory factor activates cyclooxygenase 2-prostaglandin E2 in cultured spinal microglia. Neurosci Res 2011; 71: 210-218.

18 Matsunaga J, Sinha D, Pannell L, Santis C, Solano F, Wistow GJ et al. Enzyme activity of macrophage migration inhibitory factor toward oxidized catecholamines. J Biol Chem 1999; 274: 3268-3271.

19 Matsunaga J, Sinha D, Solano F, Santis C, Wistow G, Hearing V. Macrophage migration inhibitory factor (MIF)-its role in catecholamine metabolism. Cell Mol Biol (Noisy-le-grand) 1999; 45: 1035-1040.

20 Weingarten P, Zhou QY. Protection of intracellular dopamine cytotoxicity by dopamine disposition and metabolism factors. J Neurochem 2001; 77 . 776-785.

21 Bay-Richter C, Janelidze S, Sauro A, Bucala R, Lipton J, Deierborg T et al. Behavioural and neurobiological consequences of macrophage migration inhibitory factor gene deletion in mice. J Neuroinflammation 2015; 12: 163.

22 Cobacho N, de la Calle JL, Paino CL. Dopaminergic modulation of neuropathic pain: analgesia in rats by a D2-type receptor agonist. Brain Res Bull 2014; 106: 62-71.

23 Wang N, Shen X, Bao S, Feng SW, Wang W, Liu Y et al. Dopaminergic inhibition by $\mathrm{G} 9 \mathrm{a} / \mathrm{Glp}$ complex on tyrosine hydroxylase in nerve injuryinduced hypersensitivity. Mol Pain 2016; 12: 1-10.

24 Chang Y, Zhang X, Horton JR, Upadhyay AK, Spannhoff A, Liu J et al. Structural basis for G9a-like protein lysine methyltransferase inhibition by BIX-01294. Nat Struct Mol Biol 2009; 16: 312-317.
25 Balachandran S, Rodge A, Gadekar PK, Yadav VN, Kamath D, ChetrapalKunwar A et al. Novel derivatives of ISO-1 as potent inhibitors of MIF biological function. Bioorg Med Chem Lett 2009; 19: 4773-4776.

26 Yoo CB, Jeong S, Egger G, Liang G, Phiasivongsa P, Tang C et al. Delivery of 5-aza-2'-deoxycytidine to cells using oligodeoxynucleotides. Cancer Res 2007; 67: 6400-6408.

27 Klose RJ, Zhang Y. Regulation of histone methylation by demethylimination and demethylation. Nat Rev Mol Cell Biol 2007; 8: 307-318.

28 Yoshida M, Horinouchi S, Beppu T. Trichostatin A and trapoxin: novel chemical probes for the role of histone acetylation in chromatin structure and function. Bioessays 1995; 17: 423-430.

29 Greiner D, Bonaldi T, Eskeland R, Roemer E, Imhof A. Identification of a specific inhibitor of the histone methyltransferase SU(VAR)3-9. Nat Chem Biol 2005; 1: 143-145.

30 Wiley RG, Harrison MB, Levey AI, Lappi DA. Destruction of midbrain dopaminergic neurons by using immunotoxin to dopamine transporter. Cell Mol Neurobiol 2003; 23: 839-850.

31 Bennett GJ, Xie YK. A peripheral mononeuropathy in rat that produces disorders of pain sensation like those seen in man. Pain 1988; 33: 87-107.

32 Wang X, Feng SW, Wang F, Xu S. Modeled behavior of neuropathic pain with social defect in rats: a preliminary methodology evaluation. $\mathrm{Med} \mathrm{Sci}$ Monit Basic Res 2014; 20: 164-169.

33 Randall LO, Selitto JJ. A method for measurement of analgesic activity on inflamed tissue. Arch Int Pharmacodyn Ther 1957; 111: 409-419.

34 Bradford MM. A rapid and sensitive method for the quantitation of microgram quantities of protein utilizing the principle of protein-dye binding. Anal Biochem 1976; 72: 248-254.

35 Paxinos G, Franklin K. The Mouse Brain in Stereotaxic Coordinates, Compact: The Coronal Plates and Diagrams. Academic Press: San Diego, CA, USA, 2008.

36 Xu S, Wu H, Wang X, Shen X, Guo X, Shen R et al. Tumor suppressor menin mediates peripheral nerve injury-induced neuropathic pain through potentiating synaptic plasticity. Neuroscience 2012; 223: 473-485.

37 Brakensiek K, Wingen LU, Langer F, Kreipe H, Lehmann U. Quantitative high-resolution $\mathrm{CpG}$ island mapping with Pyrosequencing reveals diseasespecific methylation patterns of the CDKN2B gene in myelodysplastic syndrome and myeloid leukemia. Clin Chem 2007; 53: 17-23.

38 Bayer Andersen K, Leander Johansen J, Hentzer M, Smith GP, Dietz GP. Protection of primary dopaminergic midbrain neurons by GPR139 agonists supports different mechanisms of $\mathrm{MPP}(+)$ and Rotenone toxicity. Front Cell Neurosci 2016; 10: 164.

39 Son HJ, Kim JY, Hahn Y, Seo SB. Negative regulation of JAK2 by H3K9 methyltransferase G9a in leukemia. Mol Cell Biol 2012; 32: 3681-3694.

40 Chang S, Chen W, Yang J. Another formula for calculating the gene change rate in real-time RT-PCR. Mol Biol Rep 2009; 36: 2165-2168.

41 Llorca-Torralba M, Borges G, Neto F, Mico JA, Berrocoso E. Noradrenergic Locus Coeruleus pathways in pain modulation. Neuroscience 2016; 338: 93-113.

42 Wang F, Shen X, Guo X, Peng Y, Liu Y, Xu S et al. Spinal macrophage migration inhibitory factor contributes to the pathogenesis of inflammatory hyperalgesia in rats. Pain 2010; 148: 275-283.

43 Gainetdinov RR, Caron MG. Monoamine transporters: from genes to behavior. Annu Rev Pharmacol Toxicol 2003; 43: 261-284.

44 Yang JW, Choi EY, Park MJ, Lee MA. Expression of tyrosine hydroxylase is epigenetically regulated in neural stem cells. Biochem Biophys Res Commun 2011; 414: 712-718.

45 Tahara T, Shibata T, Nakamura M, Yamashita H, Yoshioka D, Okubo M et al. Association between IL-17A, -17F and MIF polymorphisms predispose to CpG island hyper-methylation in gastric cancer. Int J Mol Med 2010; 25: 471-477.

46 Tachibana M, Matsumura Y, Fukuda M, Kimura H, Shinkai Y. G9a/GLP complexes independently mediate H3K9 and DNA methylation to silence transcription. EMBO J 2008; 27: 2681-2690.

47 Maze I, Covington HE 3rd, Dietz DM, LaPlant Q, Renthal W, Russo SJ et al. Essential role of the histone methyltransferase G9a in cocaine-induced plasticity. Science 2010; 327: 213-216.

48 Kreek MJ, Levran O, Reed B, Schlussman SD, Zhou Y, Butelman ER. Opiate addiction and cocaine addiction: underlying molecular neurobiology and genetics. J Clin Invest 2012; 122: 3387-3393.

49 Fujita N, Watanabe S, Ichimura T, Tsuruzoe S, Shinkai Y, Tachibana M et al. Methyl-CpG binding domain 1 (MBD1) interacts with the Suv39h1-HP1 heterochromatic complex for DNA methylation-based transcriptional repression. J Biol Chem 2003; 278: 24132-24138. 
50 Fritsch L, Robin P, Mathieu JR, Souidi M, Hinaux H, Rougeulle C et al. A subset of the histone H3 lysine 9 methyltransferases Suv39h1, G9a, GLP, and SETDB1 participate in a multimeric complex. Mol Cell 2010; 37: 46-56.

51 Koda M, Nishio Y, Hashimoto M, Kamada T, Koshizuka S, Yoshinaga K et al. Up-regulation of macrophage migration-inhibitory factor expression after compression-induced spinal cord injury in rats. Acta Neuropathol 2004; 108: 31-36.

52 Barker DJ, Root DH, Zhang S, Morales M. Multiplexed neurochemical signaling by neurons of the ventral tegmental area. J Chem Neuroanat 2016; 73: 33-42.

53 Moradi M, Yazdanian M, Haghparast A. Role of dopamine D2-like receptors within the ventral tegmental area and nucleus accumbens in antinociception induced by lateral hypothalamus stimulation. Behav Brain Res 2015; 292: 508-514.

$54 \mathrm{Li} \mathrm{AL}$, Sibi JE, Yang X, Chiao JC, Peng YB. Stimulation of the ventral tegmental area increased nociceptive thresholds and decreased spinal dorsal horn neuronal activity in rat. Exp Brain Res 2016; 234: $1505-1514$.

55 Holstege JC, Van Dijken H, Buijs RM, Goedknegt H, Gosens T, Bongers CM. Distribution of dopamine immunoreactivity in the rat, cat and monkey spinal cord. J Comp Neurol 1996; 376: 631-652.

56 Qu S, Ondo WG, Zhang X, Xie WJ, Pan TH, Le WD. Projections of diencephalic dopamine neurons into the spinal cord in mice. Exp Brain Res 2006; 168: 152-156.

57 Pappas SS, Kennedy T, Goudreau JL, Lookingland KJ. Opioid-mediated regulation of A11 diencephalospinal dopamine neurons: pharmacological evidence of activation by morphine. Neuropharmacology 2011; 61: 614-621.

58 Faramarzi G, Zendehdel M, Haghparast A. D1- and D2-like dopamine receptors within the nucleus accumbens contribute to stress-induced analgesia in formalin-related pain behaviours in rats. Eur J Pain 2016; 20: $1423-1432$.

59 Mitsi V, Zachariou V. Modulation of pain, nociception, and analgesia by the brain reward center. Neuroscience 2016; 338: 81-92.

$60 \mathrm{Wu} \mathrm{Y}, \mathrm{Na} \mathrm{X}$, Zang Y, Cui $\mathrm{Y}$, Xin W, Pang R et al. Upregulation of tumor necrosis factor-alpha in nucleus accumbens attenuates morphine-induced rewarding in a neuropathic pain model. Biochem Biophys Res Commun 2014; 449: 502-507.

61 Sagheddu C, Aroni S, De Felice M, Lecca S, Luchicchi A, Melis M et al. Enhanced serotonin and mesolimbic dopamine transmissions in a rat model of neuropathic pain. Neuropharmacology 2015; 97: 383-393.

62 Austin PJ, Beyer K, Bembrick AL, Keay KA. Peripheral nerve injury differentially regulates dopaminergic pathways in the nucleus accumbens of rats with either 'pain alone' or 'pain and disability'. Neuroscience 2010; 171: 329-343.

63 Descalzi G, Ikegami D, Ushijima T, Nestler EJ, Zachariou V, Narita M. Epigenetic mechanisms of chronic pain. Trends Neurosci 2015; 38: 237-246.

64 Machelska H, Celik MO. Recent advances in understanding neuropathic pain: glia, sex differences, and epigenetics. F1000Res 2016; 5: 2743.

65 Shinkai Y, Tachibana M. H3K9 methyltransferase G9a and the related molecule GLP. Genes Dev 2011; 25: 781-788.
66 Zhang Y, Chen SR, Laumet G, Chen H, Pan HL. Nerve injury diminishes opioid analgesia through lysine methyltransferase-mediated transcriptional repression of mu-opioid receptors in primary sensory neurons. J Biol Chem 2016; 291: 8475-8485.

67 Liang L, Zhao JY, Gu X, Wu S, Mo K, Xiong M et al. G9a inhibits CREBtriggered expression of mu opioid receptor in primary sensory neurons following peripheral nerve injury. Mol Pain 2016; 12: 1-12.

68 Laumet G, Garriga J, Chen SR, Zhang Y, Li DP, Smith TM et al. G9a is essential for epigenetic silencing of $\mathrm{K}(+)$ channel genes in acute-to-chronic pain transition. Nat Neurosci 2015; 18: 1746-1755.

69 Liang L, Gu X, Zhao JY, Wu S, Miao X, Xiao J et al. G9a participates in nerve injury-induced $\mathrm{Kcna} 2$ downregulation in primary sensory neurons. $\mathrm{Sci}$ Rep 2016; 6: 37704.

70 Rahn EJ, Guzman-Karlsson MC, David Sweatt J. Cellular, molecular, and epigenetic mechanisms in non-associative conditioning: implications for pain and memory. Neurobiol Learn Mem 2013; 105: 133-150.

71 Wang Y, Liu C, Guo QL, Yan JQ, Zhu XY, Huang CS et al. Intrathecal 5azacytidine inhibits global DNA methylation and methyl- CpG-binding protein 2 expression and alleviates neuropathic pain in rats following chronic constriction injury. Brain Res 2011; 1418: 64-69.

72 Tochiki KK, Cunningham J, Hunt SP, Geranton SM. The expression of spinal methyl-CpG-binding protein 2, DNA methyltransferases and histone deacetylases is modulated in persistent pain states. Mol Pain 2012; 8: 14.

73 Sorkin LS, Yaksh TL. Behavioral models of pain states evoked by physical injury to the peripheral nerve. Neurotherapeutics 2009; 6: 609-619.

74 Zhang J, Liang L, Miao X, Wu S, Cao J, Tao B et al. Contribution of the suppressor of variegation 3-9 homolog 1 in dorsal root ganglia and spinal cord dorsal horn to nerve injury-induced nociceptive hypersensitivity. Anesthesiology 2016; 125: 765-778.

75 Wang H, Sun H, Della Penna K, Benz RJ, Xu J, Gerhold DL et al. Chronic neuropathic pain is accompanied by global changes in gene expression and shares pathobiology with neurodegenerative diseases. Neuroscience 2002; 114: 529-546.

76 Gupta-Agarwal S, Franklin AV, DeRamus T, Wheelock M, Davis RL, McMahon LL et al. G9a/GLP histone lysine dimethyltransferase complex activity in the hippocampus and the entorhinal cortex Is required for gene activation and silencing during memory consolidation. J Neurosci 2012; 32: $5440-5453$.

(1) () () This work is licensed under a Creative Commons Attribution-NonCommercial-NoDerivs 4.0 International License. The images or other third party material in this article are included in the article's Creative Commons license, unless indicated otherwise in the credit line; if the material is not included under the Creative Commons license, users will need to obtain permission from the license holder to reproduce the material. To view a copy of this license, visit http://creativecommons.org/licenses/by-nc-nd/4.0/

(C) The Author(s) 2018

Supplementary Information accompanies the paper on Experimental \& Molecular Medicine website (http://www.nature.com/emm) 